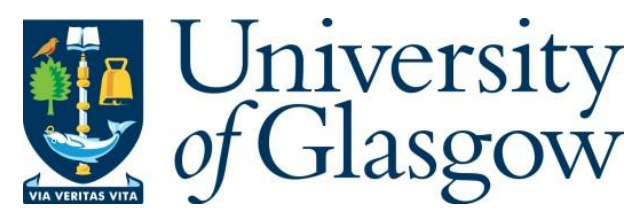

Hentze, M. W., Castello, A., Schwarzl, T. and Preiss, T. (2018) A brave new world of RNA-binding proteins. Nature Reviews Molecular Cell Biology, 19(5), pp. 327-341.

There may be differences between this version and the published version. You are advised to consult the publisher's version if you wish to cite from it.

http://eprints.gla.ac.uk/219002/

Deposited on: 12 August 2020

Enlighten - Research publications by members of the University of Glasgow http://eprints.gla.ac.uk 


\section{A Brave New World of RNA-Binding Proteins}

3 Matthias W. Hentze ${ }^{1^{*}}$, Alfredo Castello ${ }^{2}$, Thomas Schwarzl ${ }^{1}$ and Thomas Preiss $3,4^{*}$

4

5

$6 \quad{ }^{1}$ European Molecular Biology Laboratory, Meyerhofstrasse 1, 69117 Heidelberg, Germany

$7{ }^{2}$ Department of Biochemistry, University of Oxford, South Parks Road, Oxford OX1 3QU, 8 United Kingdom

$9{ }^{3}$ EMBL-Australia Collaborating Group, Department of Genome Sciences, The John Curtin

10 School of Medical Research, The Australian National University, Acton (Canberra) ACT

11 2601, Australia

$12{ }^{4}$ Victor Chang Cardiac Research Institute, Darlinghurst (Sydney), NSW 2010, Australia

13 * Correspondence to MWH (hentze@embl.de) or TP (thomas.preiss@anu.edu.au) 
$1 \quad$ O wonder!

2 How many goodly creatures are there here!

3 How beauteous mankind is! O brave new world,

$4 \quad$ That has such people in't.

5 - William Shakespeare, The Tempest, Act V, Scene I, II. 203-206 'Miranda's speech'

\section{ABSTRACT}

9 RNA-binding proteins (RBPs) are typically thought of as proteins that bind RNA through one 10 or multiple globular RNA-binding domains (RBDs) to change the fate or function of the 11 bound RNAs. Several hundreds of such RBPs have been discovered and investigated over

12 the years. Recent proteome-wide studies have more than doubled the number of proteins

13 implicated in RNA binding and uncovered hundreds of additional RBPs lacking classical

14 RBDs. This review integrates these new RBPs and discusses their possible functions, 15 including the notion that some may be regulated by RNA rather than exerting control over it.

\section{INTRODUCTION}

19 A 'classical' RNA-binding protein (RBP) participates in the formation of ribonucleoprotein

20 (RNP) complexes that are principally involved in gene expression ${ }^{1}$. It does so by binding to 21 sequence and/or structural motifs in RNA, via modular combinations of a limited set of 22 structurally well-defined RNA-binding domains (RBDs) ${ }^{2}$ such as the RNA recognition motif $23(\mathrm{RRM})^{3}$, the $\mathrm{K}$ homology $(\mathrm{KH})^{4}$ or DEAD box helicase domains (Fig. 1a) ${ }^{5}$. These assertions 24 represent decades of cumulative knowledge, including, cellular, biochemical and structural 25 data. However, recent advances in determining the structures of large RNP machines such 
1 as the ribosome $e^{6-8}$ and spliceosome ${ }^{9-11}$ reveal complex protein-RNA packaging without

2 involvement of canonical RBDs. This suggests that unorthodox RNA binding is a broader

3 phenomenon than previously anticipated.

4 A widely held assumption is that RBPs with high affinity and/or specificity for their targets

5 are more likely to have (ascertainable) biological functions ${ }^{12}$. Implicit in this conventional

6 view of RBPs is also that they should act in furtherance of the RNA's function ${ }^{13}$. A recent

7 review used the metaphor of RBPs as the mRNA's clothes that will ensure that different

8 mRNA domains (i.e. the 5' and 3' untranslated regions [UTRs] and the coding region [CDS])

9 are at times covered up or exposed, helping it to pass through different stages of its life ${ }^{14}$.

10 The processes driving change of RNP composition have been likened to those involved in

11 chromatin activity ${ }^{15,16}$. Accordingly, post-translational modifications (PTMs) of RBPs and

12 epitranscriptomic modifications of RNA, together with the action of ATP-dependent RNA

13 helicases, lead to dynamic RNP remodelling.

14 The above concepts have broad but clearly not universal applicability, as indicated by 15 emerging evidence from several directions. First, multiple microscopically visible 16 membrane-less RNP granules have been characterised in different cell types and cellular 17 compartments ${ }^{17,18}$. These include Cajal bodies and paraspeckles in the nucleus as well as 18 processing ( $\left(\mathrm{P}_{-}\right)$bodies and stress granules (SGs) in the cytoplasm. The term granule is

19 somewhat of a misnomer as several of these RNP bodies have now been shown to form by

20 liquid-liquid phase separations (LLPS), thought to be driven by intrinsically disordered 21 regions (IDRs; protein regions lacking stable tertiary structure in their native state) of their 22 constituent RBPs ${ }^{19,20}$. Their dynamicity and amorphous structure still remains puzzling, and 23 well-defined functions remain to be assigned to the formation of these RNP bodies. 24 Second, our realisation of the existence of a myriad of long noncoding RNAs (IncRNAs) 25 triggered intense efforts to uncover their functions ${ }^{21,22}$. Many IncRNAs are presently 
1 expected to act in the recruitment of transcription factors or chromatin-modifying complexes

2 to genomic loci, or otherwise organise, scaffold or inhibit protein assemblies ${ }^{23,24}$. These

3 latter scenarios tend to break with convention, indicating that an RNA may act on its protein

4 binding partner rather than itself being the target of regulation (Fig. 1b). Finally, several

5 unbiased approaches to identify RBPs proteome-wide have been developed recently,

6 yielding a growing collection of RNA-binding proteomes from multiple organisms and

7 cellular contexts. These compendia persistently identify large numbers of novel $\mathrm{RBPs}^{25}$ that

8 defy convention, lacking either discernible RBDs or established cellular functions that link

9 them to RNA biology in a straightforward fashion, or both.

10 The features and functions of classical RBPs have been expertly covered by the reviews

11 cited above and other recent works ${ }^{26-28}$. This review focuses primarily on the challenges

12 posed by unconventional RBPs, their methods of discovery and emerging information about

13 their modes of RNA-binding, RNA targets, as well as their molecular and cellular functions.

14 We will contrast and integrate these with what we know about classical RBPs.

16 THE ERA OF RNA INTERACTOMES

17 Since the discovery in the early nineties of several metabolic enzymes that engage in

18 'moonlighting' RNA-binding activity, it became apparent that the number and diverse nature

19 of RBPs had been underestimated ${ }^{29-32}$. The list of unconventional RBPs grew incrementally

20 over the decades, urging the development of methods to identify RBPs comprehensively in

21 living cells.

23 Experimental approaches to catalogue RBPs

24 In vitro approaches were devised using either immobilised RNA probes or arrayed proteins

25 (Box 1), identifying multiple novel RBPs ${ }^{33-36}$. More recently, RNA interactome capture was 
1 developed as an in vivo approach focusing on native protein-RNA interactions (Box 1). It

2 entails UV crosslinking of RBPs to RNA in live cells followed by collective capture of RNPs

3 containing poly $(\mathrm{A})^{+}$RNA on oligo(dT) beads, and identification of proteins by quantitative

4 mass spectrometry $(\mathrm{Q}-\mathrm{MS})^{37}$. Two groups independently deployed RNA interactome

5 capture to yield 860 and 791 proteins as the RBP repertoire of human HeLa and HEK293

6 cells, respectively ${ }^{38,39}$. Both RNA interactomes overlap considerably, with 543 shared

7 RBPs, and enrichment for the gene ontology (GO) term "RNA binding" (e.g. 468 of a total of

8860 proteins in the HeLa RNA interactome, based on annotation current at the time $)^{40}$, and

9 detected the majority of the well-established RBPs. Classical RBDs such as the RRM, KH,

10 DEAD box helicase and some zinc finger domains were also strongly enriched. Altogether

11 this attested to the technical robustness of the approach. Beyond that, about half of the

12 proteins in each RNA interactome lacked known RBDs, and hundreds had no previous

13 relation to RNA biology. Interestingly, both studies revealed common biological roles and

14 molecular functions among the newly discovered proteins, including intermediary

15 metabolism, cell-cycle progression, antiviral response, spindle organization, protein

16 metabolism (chaperons and prolyl cis-trans isomerases), and others ${ }^{38,39}$. The discovery of

17 RNA-binding activity of proteins involved in biological processes without apparent relation to

18 RNA biology ('enigmRBPs') suggested unexplored regulatory layers between gene

19 expression and other biological processes. About two dozen of these enigmRBPs were

20 validated by orthogonal approaches ${ }^{38,39,41}$, seven of them were analysed by

21 immunoprecipitation followed by sequencing of bound RNA, demonstrating that these

22 proteins specifically interact with distinct sets of poly $(A)^{+} \operatorname{RNAs}^{38,39}$ and exert defined

23 biological functions.

24 RNA interactome capture was since applied to samples from diverse sources (Fig. 2),

25 typically identifying several hundreds of active RBPs. Sources include several additional 
1 human $^{38,39,42-44}$ and mouse cell lines ${ }^{45-47}$, yeast ${ }^{43,48,49}$, the unicellular parasites Leishmania

2 donovani ${ }^{50}$, Plasmodium falciparum ${ }^{51}$ and Trypanosoma brucei ${ }^{52}$, as well as plants ${ }^{53-55}$,

3 flies $^{56,57}$, worms ${ }^{49}$ and fish ${ }^{58}$. The RBP sets of different origins each featured enrichment of

4 RNA-related annotation (for example, see Fig. S1a), and orthogonal methods were typically

5 used to validate some of the unexpectedly discovered RBPs, including dual-fluorescence

6 RNA-binding assay ${ }^{39,41,49}$, crosslinking and immunoprecipitation (CLIP) followed by 5'

7 radioactive labelling of RNA by T4 polynucleotide kinase $38,43,45,46,56,57$, RT-PCR ${ }^{49}$ or

8 sequencing ${ }^{38,39,43}$. Using updated annotation, we compiled here all published RNA

9 interactomes into RBP supersets for Homo sapiens (1914), Mus musculus (1393),

10 Saccharomyces cerevisiae (1273), Drosophila melanogaster (777), Arabidopsis thaliana

11 (719) and Caenorhabditis elegans (593) (Fig. 2a, Table S1). These datasets represent a

12 resource to mine for shared and selectively identified RBPs (Fig. 2b-f). While simple

13 technical reasons will explain some of the differences in coverage and overlap, the outlines

14 of shared 'core' RNA interactomes are emerging ${ }^{43}$. To illustrate this, we performed

15 InParanoid ${ }^{59,60}$ analysis to yield a high number of orthologue groups especially between

16 mammals, which expectedly decreases in more evolutionary distant organisms (Fig. $\mathbf{2 g - h}$ ).

17 On the whole, widely shared RBPs tend to be more highly enriched in established RNA-

18 related annotation than RBPs with cell or organismic context-dependent expression or

19 activity.

20 Some specific observations from these datasets deserve attention. For example, the mouse

21 embryonic stem cell (mESC) RNA interactome was found to be enriched in proteins with

22 differential expression during differentiation, suggesting that RBPs are regulated during the

23 transition from stem to differentiated cell ${ }^{45}$. In particular, the transcriptional network of the

24 proto-oncogene Myc was found to be enriched among mESC RBPs, suggesting that RBPs

25 contribute to Myc-dependent cell fate decisions. Moreover, mESC RBPs were significantly 
1 upregulated during the third day of reprogramming into induced pluripotent stem cells

2 (iPSC). RNA interactome capture was further combined with nuclear fractionation to

3 determine the repertoire of nuclear RBPs of K562 myeloid leukaemia cells; the p53

4 interaction network was enriched among the newly discovered nuclear RBPs ${ }^{61}$.

5 RNA modifying enzymes such as RNA tailing enzymes and 'writers' of epitranscriptomic

6 modifications consistently form part of RNA interactome compendia. The cardiomyocyte

7 RNA interactome contains 29 RBPs annotated for 5-methylcytosine $\left(m^{5} \mathrm{C}\right)$, N6-

8 methyladenosine $\left(\mathrm{m}^{6} \mathrm{~A}\right)$ and pseudouridine modifications, as well as adenosine-to-inosine

9 editing ${ }^{46}$. Such epitranscriptomic marks may affect RNA-protein interactions, or otherwise

10 modulate RNA function ${ }^{62,63}$ (Box 2).

11 Metabolic enzymes were recurrently identified as RBPs. Our meta-analysis revealed 71

12 such 'moonlighting' metabolic enzymes in humans, 104 in mouse and 132 in yeast RNA

13 interactomes (Table S1), adding a plethora of new examples to a list of about twenty RNA-

14 binding metabolic enzymes discovered by classic low-throughput studies ${ }^{64-66}$. Collectively,

15 these dual RBP-enzymes cover a breadth of metabolism, with interesting differences in

16 predominant pathways depending on the source material. For example, the RNA

17 interactome of Huh-7 cells harbours numerous enzymes from glycolysis and other

18 pathways of intermediary metabolism ${ }^{43,49}$. This likely relates to the important metabolic role

19 of hepatocytes at the organismal level ${ }^{43}$. The HL-1 cardiomyocyte RNA interactome exhibits

20 a high incidence particularly of mitochondrial metabolic enzymes ${ }^{46}$, reflecting their high

21 organelle content and energy needs. Many of these RNA-binding metabolic enzymes were

22 validated by orthogonal approaches ${ }^{39,43,44,46}$, including mitochondrial fractionation followed

23 by T4 polynucleotide kinase (PNK) assay ${ }^{46}$. Interestingly, some of these enzymes interact

24 with their own $m R N A^{49}$, perhaps hinting at the existence of negative feedback loops by

25 cognate enzyme-mRNA interaction under conditions of substrate or co-factor deprivation, 
1 as previously proposed ${ }^{64-68}$. Taken together, this suggests that RNA binding by metabolic

2 enzymes is a common and widely conserved occurrence.

4 In silico approaches to catalogue RBPs

5 A set of 1,542 human RBPs (7.5\% of the proteome) was defined based on computational 6 analyses that required a protein to harbour known RBDs or other domain features

7 characteristically found in proteins with RNA-related functions. This was complemented by

8 manual curation to add missing but well-documented RBPs or proteins forming part of

9 known RNPs, and to remove proteins with established RNA-unrelated functions ${ }^{69}$. Members

10 of this curated set of RBPs tend to display ubiquitous expression profiles across tissues, 11 suggesting housekeeping roles in cell biology, and the set overlaps well with the 12 experimental human RNA interactomes (Fig. S1b). Notwithstanding its strengths, the

13 approach might generate false positive results for i) proteins harbouring a classified RBD 14 that serves a different functional role in that particular case ${ }^{70}$, or ii) proteins interacting with 15 RNA solely via protein-protein interactions with a direct RBP (as in the case of Y14 in the 16 exon junction complex $)^{71}$. A similar domain/function-search algorithm was recently applied 17 to Plasmodium falciparum ${ }^{51}$. This study reported 924 RBPs, a surprisingly high fraction

$18(18.1 \%)$ of the relatively small number of protein-coding genes of the malaria parasite.

19 The propensity of RBPs to interact with other RBPs, either directly or via bridging RNAs, 20 was also exploited to identify novel $\operatorname{RBPs}^{39,72,73}$. The classification algorithm, referred to as 21 "support vector machine obtained from neighbourhood associated RBPs" (SONAR), 22 evaluates each protein of a proteome (or a dataset) against protein-protein interaction (PPI) 23 data, and calculates its RBP classification score (RCS). The algorithm was trained on sets 24 of human RBPs merged from available sources ${ }^{39,69,74}$, where the "neighbourhood" of each 25 protein was determined using the BioPlex PPI dataset ${ }^{75}$, which includes many thousands of 
1 experimentally determined protein-protein interactions. Sets of SONAR-predicted RBPs

2 were established for human (1,784 proteins), Drosophila melanogaster (489) and

3 Saccharomyces cerevisiae (745); these agree well with the experimentally determined RBP

4 sets (Fig. S1c,d). SONAR can be readily applied to any organism as long as substantial

5 PPI data are available. SONAR thus presents the same limitations as any proteomic-based

6 approach, including the depth of the protein-protein interaction data available in a given

7 organism. In addition, it may lead to false positives because proteins that interact with

8 RBPs are not always RBPs themselves ${ }^{71}$.

10 The plasticity of RNA-binding proteomes

11 Biology is dynamic. The binding of RBPs to RNA constantly changes, and the composition

12 of RNA interactomes is context-dependent and responds to stimuli. While a subset of

13 'house-keeping' RBPs might be constitutively and ubiquitously active ${ }^{69}$, many RBPs have

14 more restricted expression patterns and/or their RNA-binding activity may be regulated, e.g.

15 by post-translational modifications ${ }^{76-78}$, cofactor binding ${ }^{79}$ or protein-protein interactions ${ }^{80}$.

16 Moreover, some RBPs can 'sit idle' for lack of their RNA targets ${ }^{81-83}$. For example, cellular

17 sensors against viral infection such as interferon-induced, double-stranded RNA-activated

18 protein kinase (EIF2AK2 or PKR), retinoic acid-inducible gene-I (RIGI or DDX58) or toll-like

19 receptors (TLRs) may only be activated by the presence of unusual RNA products derived

20 from viral replication such as long double stranded RNA tracts ${ }^{81}$ or triphosphate $5^{\prime}$ ends ${ }^{82,83}$

21 (Fig. 1b).

22 RNA interactome capture has been adapted to investigate responses of RNA-binding

23 proteomes to physiological changes and environmental cues. This was recently applied to

24 murine macrophages responding to lipopolysaccharide stimulation ${ }^{47}$, primary mouse

25 embryonic fibroblasts treated with etoposide ${ }^{84}$, as well as to fruit fly and zebrafish embryos 
1 at different stages of development ${ }^{56,58}$. During the maternal-to-zygotic transition (MZT),

2 gene expression switches from maternally deposited mRNAs whose timed expression is

3 orchestrated by RBPs, to a new transcriptome emerging after zygotic genome activation

$4 \quad(Z G A)^{85}$. The dynamic changes in RBP activity expected during MZT were investigated in

5 Drosophila embryos ${ }^{56}$. Using tandem mass tags $(\mathrm{TMT})^{86}$, biological triplicate samples

6 derived from pre- (0-1 hours post fertilisation [hpf]) and post-ZGA embryos (4.5-5.5 hours)

7 were analysed. Comparison between UV-irradiated versus non-irradiated pooled pre- and

8 post-ZGA stage samples was used to determine the 'static' RNA interactome capture. In

9 separate experiments, UV-irradiated samples from pre- and post-ZGA stages were directly

10 compared for the identification of 'dynamic' RBPs. The former analysis yielded 523 proteins

11 as high confidence fly embryo RBPs, whereas the latter identified 1131 proteins, 116 of

12 which were differentially captured at $10 \%$ false discovery rate (FDR). To determine whether

13 differential RNA binding was due to alterations in protein levels, the total proteomes were

14 also determined in parallel. Comparison of the RNA interactome and whole proteome data

15 revealed three classes of RBPs: class 1 (1015 proteins) showed no significant change in

16 either RNA binding or total abundance; class 2 (78 proteins) showed commensurate

17 changes in both parameters, suggesting that differential RNA binding was due to altered

18 protein levels; and, class 3 (38 proteins) with a clear change in RNA binding without a

19 corresponding change in RBP abundance, implying a modulation in the ability of these

20 proteins to interact with RNA. These RBPs were thus dubbed 'dynamic binders'. The latter

21 include eight known splicing factors, and seven of these bind RNA more avidly in pre-MZT

22 embryos. These findings broadly concur with an analysis using mRNA expression and

23 localisation as proxies to reveal that RBP expression peaks during the prezygotic phase

24 and MZT, while transcription factors are highly expressed during the first zygotic waves and

25 mid embryogenesis ${ }^{57}$. A similarly configured analysis of zebrafish embryos before (1.75 hpf) 
1 and during zygotic genome activation (3 hpf) uncovered a repertoire of 227 RBPs active

2 during vertebrate MZT. As expected, this set included many regulators of mRNA

3 polyadenylation, translation and stability but proteins involved in RNA modification and pre-

4 mRNA processing factors were also notably represented. 24 and 53 proteins were

5 differentially active at pre-ZGA and ZGA, respectively, and appeared to be mostly 'dynamic

6 binders $^{58}$.

7 Comparative RNA interactome capture can thus be used to investigate dynamic changes in

8 RNA-binding proteomes. It should thus be feasible to study a wide range of questions, from

9 development and differentiation to signalling, metabolism, infection and other disease

10 processes and drug effects.

\section{UNORTHODOX RNA-BINDING DOMAINS}

13 Many of the RBPs newly discovered by the different methods described above lack known

14 RBDs, posing questions of how they interact with RNA. Tried-and-tested approaches exist

15 to map RBDs within individual proteins, for example by mutagenesis combined with RNA-

16 binding assays, such as electrophoretic mobility shift assay (EMSA) $)^{87}$ or the CLIP-coupled

17 PNK assay ${ }^{45}$, however, high(er) throughput methods were required to identify the RBDs of

18 hundreds of novel RBPs in a time-efficient manner. Thus, three approaches were

19 developed, each using mass-spectrometry in a different configuration to identify protein

20 regions that become crosslinked to RNA after UV exposure of live cells.

21 One approach focused on the purification and direct detection of the RNA-crosslinked

22 peptides, whose mass is altered by the nucleic acid remnant (Fig. 3a $)^{42,88}$. Data analysis

23 was performed with $\mathrm{RNP}^{\mathrm{xl}}{ }^{42}$, a custom-designed software to reduce the complexity of the

24 search for peptide-nucleotide matching spectra. Applied to yeast RBPs, RNP ${ }^{\times 1}$ identified

25376 crosslinked peptides corresponding to 133 unique crosslinking sites in 57 different 
1 proteins, mostly canonical RBPs such as ribosomal proteins and proteins with RRM or $\mathrm{KH}$

2 domains ${ }^{42}$. A number of RNA-binding sites were identified in unorthodox RBPs, including 3 the enzymes peptidyl-prolyl cis-trans isomerase (CPR1), enolase 1 (ENO1) and 4 phosphoglycerate kinase (PGK1).

5 RBDmap adds a further digestion step with a protease that cleaves every 17 amino acids 6 on average and a second round of oligo(dT) capture to the RNA interactome capture 7 workflow ${ }^{44}$. After the second oligo(dT) capture, the covalently linked polypeptides are 8 proteolysed by trypsin, generating an RNA-crosslinked peptide and a neighbouring peptide 9 with native mass (Fig 3b). RBDmap employs the neighbouring peptides with native mass to

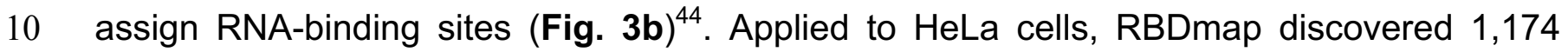
11 RNA-binding sites within 529 proteins $^{44}$, while more limited analysis of HL-1 cardiomyocytes 12 still revealed 568 RNA-binding sites from 368 proteins ${ }^{46}$. RBDmap data display strong 13 concordance with regular RNA interactome studies, reinforcing the identification of 14 unorthodox RBPs even after two oligo(dT) purification steps and after extensive proteolytic 15 treatment ${ }^{44,46}$. As expected, RBDmap "re-discovered" the classical RBDs such as the RRM$16, \mathrm{KH}-$, or cold shock domains ${ }^{2}$. Notably, many of the reported RNA-binding sites mapped to 17 globular proteins and domains lacking previous association with RNA binding. For HeLa 18 cells these include the thioredoxin (TXN) fold, heat shock protein (HSP) 70 and HSP90 19 domains, 14-3-3, domain associated with zinc fingers (DZF), PDZ and NDR (complete list $20 \mathrm{in}^{44}$ ), many of which were validated by orthogonal approaches. Interestingly, the mapped 21 RNA-binding sites showed enrichment for homologous regions across different proteins 22 from the same family, and many mapped to enzymatic cores or protein-protein interaction 23 surfaces, suggesting a possible interplay between these activities and RNA binding ${ }^{44}$. For 24 HL-1 cells, RNA-binding regions were identified in 24 metabolic enzymes, 12 of which 
1 mapped to di-nucleotide binding domains ${ }^{46}$. These data corroborate and expand the

2 previously suggested RNA-binding activity of di-nucleotide-binding domains ${ }^{32,65,66,89,90}$.

3 Unexpectedly, many of the identified RNA-binding regions mapped to IDRs, implicating

4 them as predominant sites of protein interaction with RNA in vivo (see below). Another

5 observation was that RNA-binding regions were enriched for regions mutated in Mendelian

6 diseases, while natural variants distributed equally across binding and non-binding

7 regions ${ }^{44}$. This suggests that numerous Mendelian diseases arise from altered RNA

8 biology. Finally, the RNA-binding regions strongly overlap with known post-translational

9 modification sites, including phosphorylation, acetylation and methylation ${ }^{44}$. This enrichment

10 was not observed for protein regions lacking RNA-binding activity. Thus, posttranslational

11 modifications may regulate RNA binding and RNP dynamics akin to chromatin remodelling.

12 Peptide cross-linking and affinity purification ( $\mathrm{PCLAP}$ ) is a recently described cousin of

13 RBDmap, which implements the first protease treatment directly after lysis, requiring only

14 one oligo(dT) capture round ${ }^{91}$. The trade-off is that pCLAP does not quantify the peptides in

15 the released fraction. This reference is used in RBDmap to determine with high confidence

16 the protein regions engaged in RNA binding (Fig. 3b) ${ }^{44}$.

17 A third approach, termed proteomic identification of RNA-binding regions (RBR-ID)

18 identifies peptides with reduced intensity in UV-irradiated samples compared to non-

19 irradiated controls (Fig. 3c) ${ }^{92}$. RBR-ID of nuclear proteins from mESCs detected 1,475

20 RNA-binding sites mapping to 803 mESC proteins with a 5\% FDR. $47 \%$ of the RBR-ID

21 proteins were present in the RNA interactome studies ${ }^{38,39,43,45,61}$, while 865 previously

22 known RBPs were missing. Interestingly, the 427 RBPs newly identified by RBR-ID were

23 enriched for gene-regulatory and chromatin-associated functions. At the domain level,

24 peptides with UV-dependent intensities (i.e. RNA-binding sites) mapped to RRM, KH,

25 DEAD box and other classical RBDs, validating the approach. The RNA-binding sites of the 
1427 newly discovered RBPs surprisingly often mapped to chromatin-related domains such

2 as the chromodomain and bromodomain, invoking a potential crosstalk between DNA and

3 RNA. Some of the latter results confirmed prior candidate-based approaches characterizing

4 the activity of chromatin remodelling proteins such as the catalytic subunit of the polycomb

5 repressor complex $2(\mathrm{PRC} 2) \mathrm{EZH} 2$, which has been shown to interact with long non-coding

6 RNA and nascent transcripts ${ }^{93-95}$; RBR-ID data suggest that this phenomenon might be

7 broader than previously anticipated.

8 Together, RNP ${ }^{\times 1}$, RBDmap and RBS-ID have greatly expanded the known repertoire of

9 RBDs. However, we know little about the RNA targets of these novel RBDs or the function

10 of these interactions. To address this, functional studies including determination of the

11 specificity and affinity of these novel RBDs for their target sequences ${ }^{96-100}$ will be required.

\section{NOVEL RNA-BINDING MODES}

14 The many novel RBPs and their non-typical RBDs raise pertinent questions regarding their 15 biological function(s) ${ }^{101}$. Not every bimolecular collision that happens in cells should be 16 assumed to be physiologically relevant. Which ranges for the affinities and RNA-binding 17 specificities are to be expected? Concepts regarding specificity and non-specificity in RNA18 protein interactions were recently reviewed ${ }^{12}$. A first relevant aspect is that indiscriminate

19 RNA binding by RBPs is common and can be important to their function. For example, 20 numerous proteins involved in mRNA translation (for example, the eukaryotic initiation 21 factor [elF]4F complex) and degradation need to be non-selective to fulfil their functions

22 (Fig. 4a vs b). Similarly, the exon junction complex is deposited through protein-protein

23 interactions $^{102}$ at a constant position upstream of splice junctions, and interacts with a 24 plurality of RNA sequences ${ }^{103}$ (Fig. 4c). Second, one must distinguish between 'biological 25 specificity', the binding characteristics of RBPs in vivo, and 'intrinsic specificity', such as 
1 one might determine by selection from a random pool of sequences in vitro. An interesting

2 upshot of this distinction is that intrinsically specific RBPs may function biologically as less

3 specific RBPs, when they bind their physiological RNA targets without much discrimination

4 because these do not fall into the high-affinity/high-specificity range of their RNA-binding 5 potential $^{12}$.

$7 \quad$ RNA Binding by Intrinsically Disordered Regions

8 As described above, IDRs are not only involved in aggregation of RNPs into granules by 9 protein-protein interactions ${ }^{19,20}$ but also directly engage in RNA binding ${ }^{44,46,104,105}$. RBPs are

10 enriched in IDRs that are characterized by a low content of bulky hydrophobic amino acids,

11 with the exception of tyrosine $(\mathrm{Y})$, and a high proportion of small, polar or/and charged

12 amino acids, particularly glycine $(G)$, serine $(S)$, arginine $(R)$, lysine $(K)$, glutamine $(Q)$,

13 glutamic acid (E) and aspartic acid (D) ${ }^{44}$. Interestingly, mutations in RBPs causing human

14 Mendelian diseases occur with higher frequency within these RNA-binding IDRs than in

15 globular domains, suggesting strong sequence constraints ${ }^{40}$. The occurrence of IDRs within

16 RBPs appears to be conserved from yeast to human ${ }^{43}$, often in the form of repeats such as

17 RGG, YGG, SR, DE or $\mathrm{KK}^{39,104}$. A recent report proposes that the number of repeats within

18 the IDRs of RBPs has expanded from yeast to human ${ }^{43}$. Because the number and identity

19 of globular domains present within these proteins have remained the same, IDRs may

20 represent a plastic component of RBPs that co-evolved with the increasing complexity of

21 eukaryotic transcriptomes.

22 Around half of the RNA-binding sites reported by RBDmap $(1,174)$ in HeLa cells mapped to

23 IDRs, reflecting their prevalence as a mode of RNA binding ${ }^{44} .170$ RBPs appeared to

24 interact with RNA exclusively through IDRs, suggesting that these regions can suffice to

25 mediate RNA binding. Amongst the arginine-rich motifs (ARM), RGG and SR repeats were 
1 previously reported to bind $\mathrm{RNA}^{104}$. The discovery of numerous additional examples

2 allowed assignment into sub-classes that differ by the lengths of their glycine linkers ${ }^{44,106}$.

3 Nuclear magnetic resonance (NMR) analyses of human fragile $\mathrm{X}$ mental retardation protein

4 (FMRP) showed that the positioning of the arginines is essential for selective binding of the

5 RGG box to the guanidine-rich sequence in the sc1 $\mathrm{mRNA}^{107}$, where the glycine linker

6 orients the arginines geometrically to interact with the Watson-Crick nucleotide base pairs,

7 which stack on two G-quadruplexes resulting from the protein-RNA co-folding (Fig. 4d). In

8 addition, the glycine linker contributes to RNA binding by shape complementarity

9 interactions at the interface between G-quadruplexes and Watson-Crick base pairs. Hence,

10 the affinity and selectivity of RGG boxes for their target RNAs may be determined by the

11 frequency of arginines and glycines.

12 A second RNA-binding family of IDRs involves aromatic residues, especially tyrosines (Y),

13 that combine with glycines $(G)$ and serines $(S)$ forming $[G / S] Y[G / S]$. These motifs display a

14 tendency to aggregate in vitro, inducing hydrogel formation and amyloid-like fibres, and

15 engage in dynamic liquid-liquid phase separations in vivo ${ }^{108,109}$. Aromatic residues tend to

16 form part of hydrophobic protein cores, but, when present at the protein surface, can

17 interact with amino acids or nucleotides by stacking or hydrogen bonding ${ }^{2}$. When

18 embedded in a glycine-rich context, the aromatic residue is particularly exposed, likely

19 fostering its propensity to aggregate when interacting with similar protein motifs ${ }^{108,109}$, or to

20 bind RNA ${ }^{44}$.

21 Finally, a heterogeneous set of linear motifs involving lysine $(\mathrm{K})$ and, to a lesser extent,

22 arginine $(\mathrm{R})$ was also enriched within $\mathrm{RBPs}^{44}$. Interestingly, the stoichiometry and distances

23 between the positively charged residues, as well as the combination of these with other

24 amino acids, were conserved even across non-homologous proteins. Notably, the basic

25 IDRs present in RBPs are similar to motifs in DNA-binding proteins. Here, the basic arms 
1 can alter the DNA-binding of transcription factors by a large capture radius ${ }^{110}$. In this

2 "monkey bar" model, transcription factors utilise their basic arms to reach distant DNA sites

3 by "hopping" and "sliding" instead of $3 \mathrm{D}$ diffusion ${ }^{110}$. It is currently unknown whether basic

4 arms may play similar roles in RBPs.

5 Thus, IDRs could represent malleable, potentially multifunctional RNA-binding motifs. Their

6 RNA binding can range from highly specific to non-selective, and may promote co-folding

7 upon interaction with their target RNAs ${ }^{104,105,107,111}$ (Fig. 4d). Interestingly, the high

8 sequence constraints of IDRs $^{40}$ enable RNA-binding regulation by reversible

9 posttranslational modifications such as acetylation or phosphorylation ${ }^{44}$. In principle, these

10 properties qualify IDRs as versatile modules for interaction with RNA, either alone or in

11 cooperation with globular RBDs.

13 Shape Complementarity Interactions and Protein-Binding RNAs

14 Protein-RNA interactions are typically described as a process in which a protein harbours

15 "sensors" (RBDs) to recognize and bind particular sequence and/or structural elements

16 within its "target RNA" (Fig. 1a). However, synthetic RNA aptamers can bind proteins

17 following the same molecular principles as those that enable proteins to bind $\mathrm{RNA}^{112}$,

18 suggesting that RNA can equally be the driving force mediating protein-RNA interactions

19 (Fig. 1b). The 169 annotated ribosomal proteins follow 119 distinct domain architectures ${ }^{69}$

20 but does this postulate an equal number of distinct RBDs? A more probable explanation

21 appears to be that ribosomal proteins and rRNAs have co-evolved to interact with each

22 other, where extended shape complementarity and the right spatial configuration of

23 molecular interactions play a major role in forming a perfectly packed machinery: the

24 ribosome ${ }^{8}$. Another example of intricate protein-RNA interactions is the spliceosome, where

25 small nuclear (sn)RNAs interact with proteins to form the functionally active complex ${ }^{11}$. 
1 These interactions are enabled by the ability of RNA to fold into three-dimensional (3D)-

2 structures resulting in complex surfaces with potential to interact with complementary (both

3 in shape and biochemical properties) protein partners (Fig. 1b and 4e), as illustrated by 4 tRNAs $^{113}$.

5 The fact that RNA can be more than just the "passive" partner in protein-RNA interactions is

6 also illustrated by the 5' UTRs of viral RNAs, which evolved to interact with host proteins.

7 For example, the internal ribosome entry site (IRES) of poliovirus binds the C-terminal

8 moiety of the eukaryotic initiation factor (elF)4G to recruit the $40 S$ ribosomal subunit ${ }^{114}$.

9 Similarly, the hepatitis C virus (HCV) IRES interacts directly with elF3 and the $40 \mathrm{~S}$

10 ribosomal subunit for translation initiation (Fig. 4e). A closer look at the HCV IRES-

11 ribosome co-structure shows that the interaction is not mediated by well-defined protein

12 regions endowed with RNA-binding activity; instead, the protein-RNA interface is large and

13 displays strong shape-complementarity ${ }^{115,116}$ (Fig. 4e).

14 Recently, two studies identified widespread RNA binding by chromatin-associated factors

15 and DNA-binding proteins ${ }^{61,92}$. One of the proposed possibilities is that these factors

16 interact with IncRNAs and nascent transcripts ${ }^{93-95}$. Plausibly, the IncRNAs themselves may

17 'drive' such interactions, and it might make sense to think of these RNAs as displaying

18 protein-binding activity, rather than the other way around (Fig. 1b). Functionally, the RNA

19 moiety can play different roles in this interaction, such as scaffolding protein-RNA

20 complexes as illustrated by the viral IRES of $\mathrm{HCV}^{116}$ (Fig. 4e) and the IncRNA nuclear

21 enriched abundant transcript 1 (NEAT1) in paraspeckle formation ${ }^{117}$ (Fig. 4f), or altering the

22 activity of the bound protein as exemplified by RIGI and PKR and their interaction with

23 intermediaries of viral replication ${ }^{81-83}$ (Fig. $\mathbf{4 g}$ ).

25 RNA Binding by Metabolic Enzymes 
1 RNA interactome studies have persistently identified enzymes of intermediary metabolism

2 as RBPs. Some of these enzyme RBP-RNA interactions appear to serve direct feedback

3 gene regulation. For example, thymidylate synthase (TYMS), an enzyme that catalyses the

4 formation of dTMP from dUMP, binds to its own mRNA and inhibits its translation when

5 dUMP levels are low ${ }^{118}$. A more indirect form of feedback regulation is exerted by the

6 cytosolic aconitase/iron-regulatory protein 1 (IRP1) paradigm. To be active as an enzyme,

7 the protein requires an iron sulfur cluster in its active site, which simultaneously precludes

8 RNA binding ${ }^{119}$. In iron deficiency, this cluster is lacking and, in an open conformation, IRP1

9 controls the expression of proteins to increase iron uptake and decrease its storage,

10 utilization and export ${ }^{119,120}$ (Fig. 4h).

11 Other enzymes display more oblique links to metabolism when acting as RBPs. The

12 glycolytic enzyme glyceraldehyde 3-phosphate dehydrogenase (GAPDH) oxidises its

13 substrate to generate $\mathrm{NADH}$, but it also has a diverse range of other cellular functions ${ }^{121}$.

14 An important role for GAPDH as an RBP was discovered in T lymphocyte biology ${ }^{90}$. In

15 resting $\mathrm{T}$ cells, which rely on oxidative phosphorylation for energy generation, GAPDH

16 binds to AU-rich elements (AREs) in the 3'UTRs of cytokine mRNAs including $\gamma$-interferon

17 mRNA and inhibits their translation. Following the metabolic switch to aerobic glycolysis

18 upon T cell activation, GAPDH disengages from RNA binding, thus de-repressing cytokine

19 production.

20 The high number of identified RNA-binding metabolic enzymes suggests that not all of

21 these may have 'moonlighting' functions in post-transcriptional gene regulation as

22 described above. Alternatively, (yet to be discovered) RNAs could affect their metabolic

23 function. As discussed in more detail elsewhere ${ }^{66}$, this could affect the enzyme's

24 localisation or activity, e.g. by affecting an enzymatic side reaction, by allosteric control, or

25 by providing a scaffold that organizes multi-enzyme complexes and even pathways. 
1 Interestingly, the globular Rossmann-fold (R-f) domain has emerged as a common non-

2 conventional RBD. The cardiomyocyte RNA-binding proteome includes an impressive 173

3 R-f proteins, and 29 of the 73 cardiomyocytic RNA-binding metabolic enzymes harbour at

4 least one R-f domain ${ }^{46}$. The dinucleotide-binding R-f domain of oxidoreductases has long

5 been discussed as a RNA-binding interface ${ }^{32}$. NAD+ and NADH have been reported to

6 interfere with RNA binding by GAPDH, while cytokine mRNA sequences can inhibit the

7 enzymatic activity of GAPDH in vitro ${ }^{89}$. Analysis of RBDmap data for 24 metabolic enzymes

8 (including multiple R-f domain proteins) revealed diverse spatial relationships between the

9 identified RNA contacts and previously characterized catalytically relevant regions. While

10 there is a striking overlap for some examples, in other cases the mapped RNA contacts and

11 the catalytic regions did not appear to overlap. Although this could partly reflect falsely

12 negative assignments by RBDmap, the data also implicate possible roles of RNA in

13 allosteric control or enzyme scaffolding ${ }^{46}$.

14 Many enzymes are regulated allosterically by metabolites ${ }^{66}$. Conceivably, such control

15 could also affect their RNA-binding activity. Furthermore, metabolism and metabolites could

16 control enzyme-RNA interactions via metabolite-driven PTMs. For example, S-

17 glutathionylation blocks the RNA-binding activity of GAPDH ${ }^{122}$. Many metabolic enzymes

18 are acetylated, which requires sufficient concentrations of acetyl-CoA ${ }^{123}$. More broadly,

19 RBDmap identified RBDs as hotspots for PTMs, including tyrosine phosphorylation,

20 methylation, acetylation and malonylation ${ }^{44}$. Thus, there is considerable scope for cross-talk

21 between cellular metabolism and RNA binding ${ }^{66,124}$.

\section{CHARACTERISATION OF UNORTHODOX RBPS}

24 A key step in characterising the molecular and cellular function of novel RBPs is to identify

25 their RNA targets. Several high-throughput sequencing-based methods to achieve this have 
1 emerged in recent years, either using carefully controlled in vitro methods ${ }^{125}$ or preserving

2 the context of the living cell ${ }^{126}$. Examples of the latter build on classic approaches such as

3 RNP immunoprecipitation (RIP), potentially augmented by stabilisation of complexes

4 through UV irradiation and/or chemically-induced covalent crosslinks (CLIP). These include

5 photoactivatable-ribonucleoside-enhanced and individual-nucleotide-resolution CLIP (PAR-

6 CLIP $^{97}$ and iCLIP $^{98}$, respectively). Enhanced CLIP (eCLIP; Box 1) is a variant of iCLIP with

7 improved sensitivity and specificity ${ }^{99,127}$. CLIP-type studies have already helped to

8 functionally characterise the biological roles of several unorthodox RBPs. These include

9 metabolic enzymes (e.g. HSD17B10 $)^{43}$, regulators of alternative splicing ${ }^{128,129}$, the E3

10 ubiquitin/ISG15 ligase TRIM25 ${ }^{130,131}$, the nuclear cap binding protein 3 (NCBP3, previously

11 known as C17orf85) ${ }^{132}$, the FAST kinase domain-containing protein 2 (FASTKD2) ${ }^{133}$,

12 tropomyosin ${ }^{134}$ and others.

13 The 3-hydroxyacyl-CoA dehydrogenase type-2 (HSD17B10) is a mitochondrial enzyme

14 involved in the oxidation of isoleucine, branched-chain fatty acids, and xenobiotics as well

15 as in the metabolism of sex hormones and neuroactive steroids ${ }^{135}$. Mutations in HSD17B10

16 cause a hereditary mitochondrial cardiomyopathy and neuropathy syndrome (OMIM

17 300438). Interestingly, the severity of the disorder does not correlate with the loss of

18 enzymatic activity, and the disease thus may be caused by a non-catalytic function of this

19 protein $^{136}$. A recent report identified HSD17B10 as a component of the mitochondrial

20 RNase P complex, together with mitochondrial ribonuclease P protein 1 (TRMT10C) and 3

21 (MRPP3) $)^{137}$. HSD17B10 was identified as an RBP in several RNA interactomes, indicating

22 that it binds directly to RNA ${ }^{43}$. Investigation of HSD17B10 by iCLIP $^{98}$ revealed an

23 enrichment in mitochondrial RNAs ${ }^{43}$. Specifically, HSD17B10 preferentially interacts with

24 the 5 ' ends of 15 of the 22 mitochondrial tRNAs, binding particularly at the D-stem, D-loop,

25 anticodon stem and loop regions of these tRNAs. The results suggest that a metabolic 
1 enzyme of the di-nucleotide-binding family, HSD17B10, plays a role in guiding RNase $P$ to

2 the ends of the mitochondrial tRNAs. The mutation R130C causes the classical

3 cardiomyopathy and neuropathy phenotype associated with HSD17B10 dysfunction ${ }^{136}$. This

4 mutant exhibits reduced binding to TRMT10C in vitro, although it retains the ability to form

5 tetramers ${ }^{138}$. iCLIP data revealed that the R130C mutant also displays reduced RNA-

6 binding activity ${ }^{43}$, suggesting that the dysfunctional association with RNA contributes to the

7 disease phenotype.

8 The HeLa RNA interactome catalogued four (out of six) members of the FAST kinase

9 protein family as $\mathrm{RBPs}^{39}$. One of these, the mitochondrial FASTKD2, was recurrently

10 identified in most human and mouse RNA interactomes ${ }^{38,39,44-47,61}$. Analysis of its binding

11 partners by iCLIP revealed that FASTKD2 selectively associates with mitochondrial

12 transcripts ${ }^{133}$. CRISPR-mediated depletion of FASTKD2 causes a strong reduction of its

13 binding target $16 \mathrm{~S}$ mitochondrial rRNA, in agreement with a recent report that FASTKD2 is

14 important for the assembly of the mitochondrial ribosome ${ }^{139}$. Lack of FASTKD2 function

15 also leads to a reduction in the levels of RNAs that it binds to, including those encoding the

16 complex IV components COX1, COX2 and COX3, cyb and $n d 6$ mRNAs as well as 7S RNA

17 and the prolyl-tRNA ${ }^{133}$. A nonsense mutation in FASTKD2 causes a hereditary neurological

18 disorder ${ }^{140}$. iCLIP and functional assays thus suggest that this disorder is explained by

19 defects in RNA binding causing altered mitochondrial protein synthesis and

20 metabolism ${ }^{133,139}$.

21 C17orf85 was catalogued as an RBP by RNA interactome studies ${ }^{39}$, and more recently

22 identified as a cap-binding protein that localizes to nuclear speckles, and renamed as

$23 \mathrm{NCBP}^{132}$. NCBP2 assembles with NCBP1 forming the canonical cap-binding complex

24 (CBC) that binds to nuclear RNAs and plays important roles in RNA processing and export.

25 NCBP1 knock down induced the expected retention of poly $(A)^{+}$RNA in the nucleus, and 
1 caused defects in cell proliferation ${ }^{132}$. However, almost no effect was observed when

2 NCBP2 was depleted, suggesting that NCBP2 could be replaced by another nuclear factor

3 enabled with similar cap-binding activity. NCBP3 harbours a predicted RRM and this

4 domain suffices for in vitro binding to $m^{7} G T P^{132}$. Similar to the poly(A)-specific ribonuclease

5 (PARN $)^{141}, \mathrm{NCBP3}$ interaction with $\mathrm{m}^{7} \mathrm{GTP}$ is mediated by a tryptophan and two aspartic

6 acids present at the RRM loops, and the mutation of these residues to alanine impairs the

7 cap-binding activity of $\mathrm{NCBP}^{132}$. Importantly, immunoprecipitation followed by mass

8 spectrometry revealed that, like NCBP2, NCBP3 also interacts with NCBP1 forming part of

9 an alternative nuclear cap-binding complex $(\mathrm{CBC})^{132}$.

10 Several members of the E3 ubiquitin/ISG15 tripartite motif ligase (TRIM) family have 11 recurrently been catalogued as RBPs by RNA interactome studies. This includes

12 TRIM25 $5^{39,45}$, which has additionally been validated as an RBP by orthogonal approaches

13 and its RBD was identified by RBDmap ${ }^{44,45,130}$. TRIM25 harbours an N-terminal zinc finger

14 of the RING family that is important for its ubiquitin ligase activity. Although no canonical

15 RBD can readily be recognized, TRIM25 crosslinks very efficiently to RNA ${ }^{45}$. Recently,

16 TRIM25 has been identified to interact with protein lin-28 homolog A (LIN28A) and terminal

17 uridylyltransferase 4 (TUT4), factors involved in pre-miRNA polyuridylation, enhancing their

18 activity $^{130}$. Because pre-miRNA polyuridylation triggers miRNA decay ${ }^{142}$, TRIM25 emerges

19 as a regulator of miRNA biology. TRIM25 also appears to play a role as an RBP in virus-

20 infected cells ${ }^{131}$. TRIM25 was identified as a ubiquitin ligase that triggers $\beta$-interferon ( $\beta$ -

21 IFN) through stimulation of the antiviral factor RIGI ${ }^{143}$. Notably, dengue virus 2 (DENV2)

22 genomic RNA is processed by the 5' to 3' exoribonuclease 1 (XRN1) until it gets stalled by

23 a pseudoknot present at its 3' region, leading to the production of a shorter subgenomic

24 RNA enabled with pathogenic activity ${ }^{144}$. A recent report showed that the role of this

25 subgenomic RNA is to sequester TRIM25 preventing its enhancing activity on RIGI and 
1 thus reducing $\beta$-IFN production (Fig. 5a) ${ }^{131}$. Hence, the interaction of DENV2 subgenomic

2 RNAs with TRIM25 supports its capacity to counteract the antiviral response, implicating

3 the unorthodox RBP TRIM25 in innate immunity against viruses.

4 Cyclins regulate the cell cycle by activating cyclin-dependent kinases (CDK). They have 5 also sporadically been identified as unconventional RBPs in RNA interactome capture 6 studies ${ }^{38,45,46,56}$. The Cyclins B and T are part of the RNA interactome of Drosophila

7 embryos, and Cyclin B RNA-binding activity was validated by CLIP-PNK assay ${ }^{56}$. Cyclins

8 (CCN) A2, L1 and T1 were identified in the nuclear RNA-binding proteome of mouse

9 embryonic stem cells ${ }^{92}$. Mice with reduced CCNA2 expression are prone to tumour

10 formation and chromosomal instability due to a predisposition to form lagging chromosomes

11 and chromatin bridges ${ }^{145}$. This defect resulted from insufficient expression of the meiotic

12 recombination 11 (Mre11) nuclease, apparently due to impaired translation. CCNA2 directly

13 binds to two evolutionarily conserved regions in the 3'UTR of Mre11 mRNA, which appears

14 to be necessary and sufficient for CCNA2 regulation (Fig. 5b) $)^{145}$. A C-terminal CCNA2

15 fragment lacking CDK-binding is both necessary and sufficient for RNA-binding, and its

16 expression in mouse embryonic fibroblasts restored appropriate Mre11 synthesis.

17 Interestingly, the CCNA2 RNA-interacting region binds elF4A2. Taken together, the data

18 identify an unexpected, CDK-independent function of CCNA2 as an RNA-binding protein to

19 promote Mre11 mRNA translation, potentially through an interaction with elF4A2.

\section{OUTLOOK}

22 What do we have to expect with so many new RBPs to be considered? Some might side

23 with Miranda from Shakespeare's Tempest and marvel at these novel and goodly RBPs

24 that populate the RNA interactome. Others may fear dystopia, as presented by Aldous

25 Huxley's in his novel 'Brave New World', where newly discovered RBPs represent 
1 nonconformist misfits lacking biological function. Which roles do these new RBPs play?

2 Some may indeed play none, having been discovered on the basis of a biophysical property

3 that mediates above background interaction with RNA without biological consequence for

4 the protein or the RNAs that it interacts with. Quite remarkably, however, the list of

5 unorthodox RBPs that fulfil professional roles continues to grow, as discussed above.

6 Although better known for other biological functions and lacking in classical RBDs, they

7 'moonlight' as RBPs and affect RNA fate, akin to the functions of orthodox RBPs (Fig. 1a).

8 It will be illuminating to study these RNA-protein interactions structurally. Likewise, it will be

9 important to decipher the mechanisms by which these RNA-protein interactions are

10 regulated, both by epitranscriptomic changes as well as by posttranslational modifications.

11 An intriguing facet of this is the question of whether and how intrinsically disordered regions

12 that bind RNA contribute to the formation of higher order assemblies by liquid-liquid phase

13 separation and to understand the role of RNA in these transitions.

14 Might unorthodox RBPs be controlled by RNA? We have become accustomed to the view

15 that protein functions are typically modulated by other proteins, but there is ample room for

16 the possibility that the known biological function of a protein be altered by 'riboregulation', a

17 change in the protein's function elicited by interaction with RNA (Fig. 1b). The RBPs PKR

18 and RIGI can serve as examples of this class of proteins that could vastly expand. We

19 already know much about RBPs, but future experiments are bound to surprise our intuition.

\section{ACKNOWLEDGEMENTS}

23 We dedicate this review to the memory of Bernd Fischer, who sadly passed away while this 24 review was in preparation. 
1 The authors are grateful to the members of their laboratories for helpful discussions

2 throughout. The authors also acknowledge research funding from the European Research

3 Council ERC-2011-ADG_20110310 (MWH), Medical Research Council Career

4 Development Award MR/L019434/1 (AC), the EMBL Interdisciplinary Postdocs 2

5 EIPOD2/291772 program (TS) and the National Health and Medical Research Council 6 APP1120483 (MWH and TP).

\section{Box 1. Technical approaches to study RNA-protein interactions}

System-wide identification of RBPs in vitro: In one approach, a series of immobilised RNA probes are used as bait, exposed to cellular extracts and binding proteins are identified by quantitative mass spectrometry (Q-MS) (panel a $)^{33}$. Applied to combinations of different mRNA UTRs and human HeLa extract, this yielded a dozen proteins that differentially bound to the baits, several of these were novel RBPs. In a more recent iteration, incubation of a set of pre-miRNA baits with multiple different cell lysates yielded $\sim 180$ RBPs with distinct specificities ${ }^{36}$. In a second approach, arrayed proteins are provided as bait and incubated with labelled cellular RNA. RNA binding is determined by measuring the fluorescence intensity at each individual protein spot, in analogy to DNA microarrays (panel b). Two such proteome-wide screens identified $180^{34}$ and $68^{35}$ yeast RBPs, respectively. In a third approach, purified polyadenylated $\left[\operatorname{poly}(A)^{+}\right]$cellular RNA was immobilized on oligo(dT) magnetic beads and, after incubation with cell extract, bound proteins were analysed by Q-MS ${ }^{35} .88$ mostly highly abundant proteins were identified and 22 were known RBPs.

Identification of in vivo RBP repertoires by RNA interactome capture. In this approach, UV crosslinking of cultured cells or organisms covalently links proteins to RNA positioned at "zero distance". This is followed by denaturing cell lysis, collective capture of all RNPs 
1 involving poly $(\mathrm{A})^{+}$RNA on oligo(dT) beads, and identification of proteins by quantitative mass spectrometry (Q-MS) ${ }^{37}$ (panel c). One study used both conventional crosslinking (cCL) and photoactivatable ribonucleoside-enhanced crosslinking (PAR-CL) ${ }^{97}$. The first relies on the natural excitability of nucleoside bases by $254 \mathrm{~nm}$ ultraviolet light, which generates short-lived, free radicals that attack amino acids in close proximity forming covalent bonds ${ }^{146}$. By contrast, PAR-CL utilizes the nucleoside analogue 4-thiouridine (4SU), which is taken up by cultured cells and incorporated into nascent RNAs.

Crosslinking is then achieved by irradiation with ultraviolet light at $365 \mathrm{~nm}^{97}$. Another study also used PAR-CL, combining 4SU and 6-thioguanosine (6SG) labelling ${ }^{38}$. This latter study further exploited the $U$ to $C$ transitions occurring as a consequence of the crosslinking between $4 S U$ and the RBP to analyse globally the footprints of the RNA interactome on the RNA. The protocol has been adapted to different model systems ${ }^{43,48,49,53-57}$, can be used to monitor differential association of RBPs with RNA under different physiological conditions or in response to biological cues $^{47,56}$ as well as to identify RBPs in different subcellular compartments $^{61}$.

The eCLIP method. This approach is used to determine the footprints of a given RBP on its targets RNA with single nucleotide resolution ${ }^{99,127}$. UV-irradiation of live cells is followed by cell lysis and limited RNA digestion to fragment RNA. Protein-RNA complexes are immunoprecipitated with an antibody against the RBP under study. Then, the immunoprecipitated material is resolved by denaturing gel electrophoresis, transferred to a membrane. Segments of the membrane corresponding to RNPs are excised. The RNA is recovered and after cDNA library, the RNA regions bound by the RBP are identified by high throughput sequencing (panel d). Since reverse-transcription often stalls at the site of the protein-RNA crosslink, eCLIP affords single nucleotide resolution. TAG-eCLIP broadens the scope of the approach, as it includes a CRISPR/Cas9-mediated insertion of a C-terminal 
1 affinity tag into the endogenous RBP gene, bypassing the need for individual antibodies ${ }^{147}$. Together with several hundred antibodies against known RBPs that have now been tested for immunoprecipitation ${ }^{74}$, this provides for a growing list of eCLIP datasets, which are accessible at https://www.encodeproject.org. Among the 122 proteins with available eCLIP data, 34 lack classical RBDs.

Box 2. Epitranscriptomic control of RNA binding. Progress has been swiftest with understanding $\mathrm{m}^{6} \mathrm{~A}$ function in diverse cellular contexts. The YT521-B homology (YTH) domain proteins were identified as $\mathrm{m}^{6} \mathrm{~A}$ 'readers' that specifically bind $\mathrm{m}^{6} \mathrm{~A}$-containing mRNA regions to impact splicing, export, translation, or turnover ${ }^{62,63}$. The ensuing downstream effects are determined by the specific YTH protein that is recruited and on the mRNA context. The YTH-containing proteins YTHDC1, YTHDC2, YTHDF1, YTHDF2 and YTHDF3 were consistently found in both human and mouse RNA interactome datasets. The RNA-binding proteome of $A$. thaliana contains most plant YTH domain proteins, including cleavage and polyadenylation specificity factor 30 (AtCPSF30), possibly explaining a plant-specific link between $\mathrm{m}^{6} \mathrm{~A}$ and mRNA cleavage ${ }^{54}$. When YTHDF1 is recruited to $\mathrm{m}^{6} \mathrm{~A}$ sites in the $3^{\prime} \mathrm{UTR}$ of human mRNAs, it enhances their translation, likely through interactions with subunits of the translation initiation factor elF3 ${ }^{148}$. Conversely, when YTHDF2 binds to 5'UTR sites in the nucleus of murine embryonic fibroblasts, it blocks their demethylation in heat shock, thus facilitating selective translation in the cytoplasm by direct binding of elF3 to these $\mathrm{m}^{6} \mathrm{~A}$ sites $^{149}$. More generally, cytoplasmic YTHDF2 binds $\mathrm{m}^{6} \mathrm{~A}$-containing mRNAs in human cells and promotes their relocation to processing bodies and degradation ${ }^{150}$. The latter function is critically involved in murine embryonic stem cell differentiation $^{151-153}$, facilitates maternal mRNA clearance, and the maternal-to-zygotic transition in zebrafish embryos ${ }^{154}$. Epitranscriptomic marks can also modulate RBP binding 

indirectly by affecting RNA structure. For example, the nuclear protein HNRNPC responds to $\mathrm{m}^{6} \mathrm{~A}$-operated 'structural switches' to gain access to thousands of its target sites in human nuclear RNAs ${ }^{155}$. HNRNPC preferentially binds to single-stranded $\mathrm{U}$ tracts, and $\mathrm{m}^{6} \mathrm{~A}$ can destabilise local RNA structure, making the U-tracts more accessible. Thus, when considering the determinants of RNP formation, epitranscriptomic changes need to be considered in addition to posttranslational RBP modifications. 
3 Figure 1. Functional crosstalk between proteins and RNA. Schematic representation of

4 the biological consequences of protein-RNA interactions. (a) RNA-binding proteins (RBP)

5 interact with RNA through defined RNA-binding domains to regulate various aspects of the

6 RNA's function. (b) Inversely, the RNA binds to the RBP to affect the protein's fate.

8 Figure 2. Comparison of published RNA interactomes. The reported lists applying the

9 most stringent criteria for RBPs identified by different studies and from various source

10 materials were extracted, curated and their annotations were updated, and converted to

11 base identifiers. The underlying data are provided in Table S1. (a) Supersets of RBPs

12 identified by the combination of RBP detection studies in a given organism. These were

13 compiled for Mus musculus (Mm), Homo sapiens (Hs), Saccharomyces cerevisiae (Sc),

14 Drosophila melanogaster (Dm), Arabidopsis thaliana (At) and Caenorhabditis elegans (Ce).

15 RBP sets for Danio rerio (Dr), Trypanosoma brucei (Tb), Leishmania donovani (Ld) and

16 Plasmodium falciparum (Pf) were also added. (b-h) Venn Diagrams and UpSet plots ${ }^{156}$

17 showing overlaps, set and intersections sizes between different RBP sets. Differences in

18 technical approaches and data processing (for example, thresholds for detection and

19 reproducibility) will affect these comparisons. (b) RBP repertoires as detected in human

20 source material. RNA interactome capture (RIC; Box 1) with either conventional UV

21 crosslinking $(\mathrm{CCL})$ or photoactivatable ribonucleoside-enhanced crosslinking (PAR-CL) was

22 applied to the following cell lines: cervical cancer HeLa ${ }^{39}$, embryonic kidney HEK293 ${ }^{38,39}$,

23 hepatocytic $\mathrm{HuH}^{43}$ and myeloid leukaemia K562 (serial nuclear RIC) ${ }^{61}$. HeLa cells were

24 further subjected to RBDmap ${ }^{44}$ and $\mathrm{RNP}^{\mathrm{x} \mid 42}$. The human datasets show very high overlap,

25 likely because of the preponderance of 'generic' cell lines as source material and related 
1 experimental approaches. (c) Murine RBP repertoires. RIC was applied to primary

2 embryonic fibroblasts (MEF \pm etoposide) ${ }^{84}$, embryonic stem cells (mESC, total cell and 3 nuclear fraction), macrophages (RAW264.7 \pm lipopolysaccharide) ${ }^{47}$. HL-1 cardiomyocytes

4 were subjected to both RIC and RBDmap ${ }^{44}$. These datasets overlap less well, probably due

5 to the use of more 'idiosyncratic' cell lines and inclusion of drug treatments. (d) Budding

6 yeast RNA-binding proteomes. Two studies used either in vitro protein arrays or, in one

7 case, oligo(dT) capture screens to identify RBPs ${ }^{34},{ }^{35}$. Three in vivo RNA interactomes were

8 generated, either employing conventional UV crosslinking $(\mathrm{CCL})^{48,49}$ or photoactivatable

9 ribonucleoside-enhanced crosslinking $(\mathrm{PAR}-\mathrm{CL})^{43}$. $\mathrm{RNP}^{\mathrm{xl}}$ was used with two crosslinking

10 approaches ${ }^{42}$. The diversity of technical approaches likely explains the big differences in

11 coverage and overlap. (e) Fruit fly RBP sets. Two studies applied RNA interactome capture

12 to Drosophila melanogaster embryos, using solely $\mathrm{CCL}^{56}$ or both $\mathrm{CCL}$ and $\mathrm{PAR}-\mathrm{CL}^{57}$. The

13 focus here were embryos undergoing maternal-to-zygotic transition. This, together with

14 differences in mass spectrometry approaches likely underlies the moderate overlap. (f)

15 RNA interactomes captured from different plant sources, including cell suspension cultures

16 and leaves ${ }^{53}$, etiolated seedlings ${ }^{54}$ and leaf mesophyll protoplasts ${ }^{55}$. Given the

17 heterogeneous sources, the three datasets agree reasonably well with each other. The

18 lower RBP identification rates suggest UV crosslinking limitations, likely due to the

19 presence of a cell wall and/or UV-absorbing pigments. (g) Pairwise comparisons of

20 InParanoid clusters between human $(\mathrm{Hs})$, mouse $(\mathrm{Mm})$ and yeast $(\mathrm{Sc})$. The intersections

21 between these sets constitute emerging mammalian or eukaryotic 'core' interactomes. (h)

22 UpSet plot showing the overlap between the human superset of RBPs and human

23 orthologues from Mus musculus (Mm), Saccharomyces cerevisiae (Se), Drosophila

24 melanogaster (Dm), Caenorhabditis elegans (Ce) and Arabidopsis thaliana (At). 
1 Figure 3. System-wide, high resolution identification of RNA-binding domains. Each

2 of the three methods uses UV irradiation of live cells to establish covalent bonds at direct

3 contact sites between RNA and protein (indicated by a star). Following cell lysis, the

4 approaches diverge with regard to proteolysis, purification strategy and detection by

5 quantitative mass spectrometry (Q-MS). (a) Purification and direct detection of RNA-

6 crosslinked tryptic peptides ${ }^{42,88}$. Although simple in principle, this strategy is challenging

7 due to the inefficiency of UV crosslinking and the heterogeneous mass contribution by the

8 nucleic acid remnant, resulting in sub-stoichiometric amounts of peptides with hard-to-

9 predict additional mass. To overcome this, covalently linked protein-RNA complexes are

10 purified on oligo(dT) beads, by performing the initial steps of RNA interactome capture ${ }^{37,43}$.

11 After digestion with trypsin and RNases, peptides crosslinked to remnants of RNA are

12 further enriched using a $\mathrm{TiO}_{2}$ matrix ${ }^{157}$, prior to analysis by Q-MS and a complex search,

13 performed by a custom-designed software termed $\mathrm{RNP}^{\mathrm{xl}}$, for peptide spectra with a defined

14 mass shift caused by the nucleotide remnant. (b) Extrapolation of RNA-protein crosslink

15 sites by RBDmap ${ }^{44}$. Again, RBPs are purified with poly $(\mathrm{A})^{+}$RNA as per RNA interactome

16 capture $^{37,43}$, but then digested with a protease that cleaves every 17 amino acids on

17 average (LysC or ArgC), typically leaving peptides that still contain an internal trypsin

18 cleavage site. RNA-linked peptides, termed RBDpep, are then recaptured on oligo(dT)

19 beads, while those distant to the crosslink site are released into the supernatant. Both

20 fractions are digested with trypsin and analysed by Q-MS. For RNA-bound material this

21 leads to: i) a fragment with remnant RNA that will not be identified; and ii) a neighbouring

22 fragment(s) with native mass (N-peptide). N-peptides that are enriched in the RNA-bound

23 fraction are extended in silico to the next LysC/ArgC cleavage site to reconstitute the

24 original RBDpep. (c) Proteomic identification of RNA-binding regions (RBR-ID) ${ }^{92}$. This

25 approach directly exploits the mass shift of RNA-crosslinked peptides in conventional MS 
1 analyses, by assigning RNA-binding activity to tryptic peptides ( $\sim 9$ amino acid length on

2 average) with reproducibly reduced ion counts (peptide intensity) in UV-irradiated samples 3 compared to non-irradiated controls.

4 The three approaches have some common limitations. UV crosslinking relies on a 5 favourable geometry, nucleotide and amino acid composition at protein-RNA interfaces.

6 Protein-RNA interactions with the phosphate backbone, for example, are non-optimal in this 7 regard, and will be missed. Mass-spectrometric analyses are also influenced by the 8 abundance of the peptides and their amino acid sequence (influencing optimal peptide size 9 generated derived from tryptic cleavage and optimal mass-to-charge ratio). The RNP ${ }^{\mathrm{xl}}$ 10 workflow offers the unique advantage of single amino acid resolution, but at the expense of 11 a limited sensitivity and the need for specialized proteomic analyses. RBDmap is less 12 complex to implement and more sensitive, but at the price of a lower resolution ( $\sim 17$ amino 13 acids). The conceptually very straightforward RBR-ID gives an intermediate resolution ( $\sim 9$ 14 amino acids), however, its high intra- and inter-experimental variability call for a sufficiently 15 high number of technical and biological replicates to obtain high confidence results.

17 Figure 4. Modes of RNA binding. (a) Schematic representation of a RBP harbouring a 18 classic RBD (RRM) interacting specifically with a specific RNA sequence in the context of a 19 stem-loop 2 . (b) Depiction of the eukaryotic initiation factor (elF)4F, which is composed of 20 the cap-binding protein elF4E, elF4G and the helicase elF4A. This complex associates with 21 capped RNA in a sequence-independent manner to enable global initiation of translation ${ }^{158}$.

22 (c) The exon junction complex is deposited by its interaction with CWC22 20 nucleotides 23 upstream the exon-exon junction immediately after intron removal ${ }^{102}$. (d) The disordered 24 RGG-motif present in FMR1 protein co-folds with its target RNA forming a tight electrostatic 25 and shape-complementarity driven interaction ${ }^{107}$. (e) The internal ribosome entry site 
1 (IRES) of hepatitis $\mathrm{C}$ virus (HCV), interacts directly with the ribosome through a complex

2 interaction mode that involves shape-complementarity ${ }^{116}$. (f) The IncRNA NEAT1 hijacks

3 the RBPs NONO, PSC1 and SFPQ in paraspeckles ${ }^{117}$. (g) PKR binds to double-stranded

4 (ds)RNA, derived from viral replication. RNA binding promotes protein dimerization and

5 autophosphorylation, activating PKR. In its active form, PKR phosphorylates elF2 $\alpha$ to block

6 protein synthesis in infected cells ${ }^{159}$. (h) Aconitase 1 (ACO1) associates with an iron-

7 sulphur cluster to catalyse the interconversion between citrate and isocitrate. Under low iron

8 conditions, the iron-sulphur cluster is no longer synthesised and the apoprotein, iron

9 regulatory protein 1 (IRP1), binds mRNAs encoding cellular factors involved in iron

10 homeostasis, regulating their fate ${ }^{119}$.

12 Figure 5. Examples of the biological roles of unorthodox RBPs. (a) Schematic

13 representation of (a) TRIM25 hijacking by the subgenomic RNA of DENV to reduce $\beta$ -

14 interferon ( $\beta$-IFN) synthesis ${ }^{131}$. DENV genomic RNA degradation by XRN1 leads to the

15 generation of a subgenomic RNA due to XRN1 stalling at a pseudoknot present at the 3'

16 region of this RNA. The subgenomic DENV RNA recruits TRIM25 but not RIGI, which

17 requires the presence of tri-phosphate 5' ends to interact, thus hijacking TRIM25 in RNPs

18 lacking its molecular partner in the $\beta$-IFN pathway, RIGI. (b) Cdk-independent function of

19 CCNA2 as an RNA-binding protein. CCNA2 directly binds to two evolutionarily conserved

20 regions in the 3'UTR of Mre11 mRNA and can to promote Mre11 mRNA translation,

21 potentially through an interaction with elF4A2 $2^{145}$.

\section{GLOSSARY}

24 Ribonucleoprotein (RNP): A complex formed between one or several RNAs and proteins. 
1 RNA-binding domain (RBD): A subdomain within a protein that mediates direct interaction

2 with RNA.

3 RNA-recognition motif (RRM): One of the most common RBDs, consisting of $\sim 90$ amino

4 acids that fold into two $\alpha$-helices packed against a four-stranded $\beta$-sheet. Typically, the 5 residues in the $\beta$-sheet interact with RNA.

6 HnRNP K homology $(\mathrm{KH})$ domain: Another common RBD, consisting of $\sim 70$ amino acids

7 that fold into three $\alpha$-helices packed against a three-stranded $\beta$-sheet. RNA binds to a

8 hydrophobic cleft formed between two core $\alpha$-helices and a GXXG loop that interconnects

9 them.

10 DEAD box protein: RNA helicases exhibiting two highly similar domains that resemble the

11 bacterial recombinase A. ATP binds in a cleft between the two helicase domains, while

12 RNA binds across both domains on the opposite side. The DEAD box is one of nine

13 conserved sequence motifs in this class of proteins and contains the amino acid sequence

14 Asp-Glu-Ala-Asp (DEAD).

15 Epitranscriptome: A collective term for all chemically diverse RNA modifications that exist

16 within a transcriptome. In analogy to the epigenome, the term further implies that many

17 such modifications (e.g. methylation, pseudouridylation) are deliberately placed and serve

18 regulatory roles.

19 Maternal-to-zygotic transition (MZT): The phase in embryonic development during which

20 control by maternally derived products ceases and the zygotic genome becomes activated.

21 Processing (P-)bodies: Microscopically visible foci present in the cytoplasm of eukaryotic

22 cells. P-bodies contain mRNAs and many components of mRNA silencing and turnover.

23 Stress granules (SGs): Cytoplasmic aggregates of stalled translation initiation complexes

24 in eukaryotic cells that are induced by different forms of cellular stress. 
1 Liquid-liquid phase separations (LLPS): A (bio-)physical process whereby non-

2 membrane-bound compartments (e.g. SGs, P-bodies) are formed as phase-separated,

3 liquid-like droplets within cells.

4 Intrinsically disordered regions (IDRs): Features within a native protein that lack stable

5 secondary or tertiary structure and thus appear unfolded.

6 Long noncoding RNAs (IncRNAs): RNAs longer than 200 nucleotides without ascribed

7 protein-coding potential. The arbitrary length criterium is used to distinguish IncRNAs from

8 small noncoding RNA types, such as e.g. microRNAs or tRNAs.

9 UV crosslinking: A method using ultraviolet light irradiation to covalently connect proteins

10 and RNA when positioned in very close proximity ('zero distance') to each other. UV

11 crosslinking can be applied in vitro or in living cells.

12 Zinc finger domain: A protein domain involving cysteines $(C)$ and histidines $(H)$ that

13 coordinates zinc cations. Zinc finger domains are classified based on the order and

14 frequency of $\mathrm{C}$ and $\mathrm{H}$, and can mediate interactions with DNA, RNA or proteins depending

15 on the subclass.

16 InParanoid analyses: Method for detecting orthologues and in-paralog clusters across

17 different, often distant species.

18 BioPlex PPI dataset: Comprehensive collection of protein-protein interaction networks

19 generated by experimental approaches.

20 Electrophoretic mobility shift assay (EMSA): A method to study protein interactions with

21 nucleic acids in vitro. Cell extracts or purified proteins are incubated with radiolabelled (or

22 fluorescently labelled) nucleic acids, and the resulting complexes are resolved through a

23 native gel. If the nucleic acid is bound by a protein, the protein will retard its mobility through

24 the gel compared to the unbound probe. 
1 G-quadruplex: RNA structure involving two or more stacks formed by a planar array of four

2 guanine bases $(G)$. It requires the coordination of a monovalent cation of an appropriate

3 radius, such as potassium.

4 RNA aptamer: Relatively short and often highly folded short RNA molecule that was 5 experimentally selected for specific, high affinity interactions with proteins or other 6 molecules.

7 RNase P complex: Ribonuclease complex for processing of precursor t-RNA

8 Rossmann-fold (R-f): A protein domain characterised by the presence of up to seven

9 mostly parallel $\beta$-strands combined with connecting $\alpha$-helices. This domain is typically found

10 in proteins that bind nucleotides, such as metabolic enzymes with di-nucleotide (NAD,

11 NADP, FAD) binding activity.

12 Uridylation: A biochemical reaction mediated by uridylyltransferases (e.g. TUTase) that

13 involves the addition of multiple uridines to the 3' end of RNA molecules. This modification

14 is typically a signal for RNA degradation.

\section{REFERENCES}

1 Dreyfuss, G., Kim, V. N. \& Kataoka, N. Messenger-RNA-binding proteins and the messages they carry. Nat Rev Mol Cell Biol 3, 195-205, (2002). Lunde, B. M., Moore, C. \& Varani, G. RNA-binding proteins: modular design for efficient function. Nat Rev Mol Cell Biol 8, 479-490, (2007).

233 Clery, A., Blatter, M. \& Allain, F. H. RNA recognition motifs: boring? Not quite. Curr Opin Struct Biol 18, 290-298, (2008).

254 Valverde, R., Edwards, L. \& Regan, L. Structure and function of KH domains. FEBS $26 \quad J$ 275, 2712-2726, (2008).

275 Linder, P. \& Jankowsky, E. From unwinding to clamping - the DEAD box RNA helicase family. Nat Rev Mol Cell Biol 12, 505-516, (2011).

6 Ramakrishnan, V. The ribosome emerges from a black box. Cell 159, 979-984, (2014).

7 Steitz, T. A. A structural understanding of the dynamic ribosome machine. Nat Rev Mol Cell Biol 9, 242-253, (2008). 
8 Behrmann, E., Loerke, J., Budkevich, T. V., Yamamoto, K., Schmidt, A., Penczek, P. A., ... Spahn, C. M. Structural snapshots of actively translating human ribosomes. Cell 161, 845-857, (2015).

9 Matera, A. G. \& Wang, Z. A day in the life of the spliceosome. Nat Rev Mol Cell Biol 15, 108-121, (2014).

10 Papasaikas, P. \& Valcarcel, J. The Spliceosome: The Ultimate RNA Chaperone and Sculptor. Trends Biochem Sci 41, 33-45, (2016).

11 Plaschka, C., Lin, P. C. \& Nagai, K. Structure of a pre-catalytic spliceosome. Nature 546, 617-621, (2017).

12 Jankowsky, E. \& Harris, M. E. Specificity and nonspecificity in RNA-protein interactions. Nat Rev Mol Cell Biol 16, 533-544, (2015).

13 Gebauer, F., Preiss, T. \& Hentze, M. W. From cis-regulatory elements to complex RNPs and back. Cold Spring Harb Perspect Biol 4, a012245, (2012).

14 Singh, G., Pratt, G., Yeo, G. W. \& Moore, M. J. The Clothes Make the mRNA: Past and Present Trends in mRNP Fashion. Annu Rev Biochem 84, 325-354, (2015).

15 Lee, S. R. \& Lykke-Andersen, J. Emerging roles for ribonucleoprotein modification and remodeling in controlling RNA fate. Trends Cell Biol 23, 504-510, (2013).

16 Chen, C. Y. \& Shyu, A. B. Emerging mechanisms of mRNP remodeling regulation. Wiley Interdiscip Rev RNA 5, 713-722, (2014).

17 Anderson, P. \& Kedersha, N. RNA granules: post-transcriptional and epigenetic modulators of gene expression. Nat Rev Mol Cell Biol 10, 430-436, (2009).

18 Buchan, J. R. mRNP granules. Assembly, function, and connections with disease. RNA Biol 11, 1019-1030, (2014).

19 Protter, D. S. \& Parker, R. Principles and Properties of Stress Granules. Trends Cell Biol 26, 668-679, (2016).

20 Wright, P. E. \& Dyson, H. J. Intrinsically disordered proteins in cellular signalling and regulation. Nat Rev Mol Cell Biol 16, 18-29, (2015).

21 Gloss, B. S. \& Dinger, M. E. The specificity of long noncoding RNA expression. Biochim Biophys Acta 1859, 16-22, (2016).

22 Geisler, S. \& Coller, J. RNA in unexpected places: long non-coding RNA functions in diverse cellular contexts. Nat Rev Mol Cell Biol 14, 699-712, (2013).

23 Hudson, W. H. \& Ortlund, E. A. The structure, function and evolution of proteins that bind DNA and RNA. Nat Rev Mol Cell Biol 15, 749-760, (2014).

24 Cech, T. R. \& Steitz, J. A. The noncoding RNA revolution-trashing old rules to forge new ones. Cell 157, 77-94, (2014).

25 Beckmann, B. M., Castello, A. \& Medenbach, J. The expanding universe of ribonucleoproteins: of novel RNA-binding proteins and unconventional interactions. Pflugers Arch 468, 1029-1040, (2016).

26 Gehring, N. H., Wahle, E. \& Fischer, U. Deciphering the mRNP Code: RNA-Bound Determinants of Post-Transcriptional Gene Regulation. Trends Biochem Sci, (2017).

27 Rissland, O. S. The organization and regulation of mRNA-protein complexes. Wiley Interdiscip Rev RNA 8, (2017).

28 Muller-McNicoll, M. \& Neugebauer, K. M. How cells get the message: dynamic assembly and function of mRNA-protein complexes. Nat Rev Genet 14, 275-287, (2013).

29 Hentze, M. W. \& Argos, P. Homology between IRE-BP, a regulatory RNA-binding protein, aconitase, and isopropylmalate isomerase. Nucleic Acids Res 19, 17391740, (1991). 
Rouault, T. A., Stout, C. D., Kaptain, S., Harford, J. B. \& Klausner, R. D. Structural relationship between an iron-regulated RNA-binding protein (IRE-BP) and aconitase: functional implications. Cell 64, 881-883, (1991).

31 Chu, E., Voeller, D., Koeller, D. M., Drake, J. C., Takimoto, C. H., Maley, G. F., . . . Allegra, C. J. Identification of an RNA binding site for human thymidylate synthase. Proc Natl Acad Sci U S A 90, 517-521, (1993).

32 Hentze, M. W. Enzymes as RNA-binding proteins: a role for (di)nucleotide-binding domains? Trends Biochem Sci 19, 101-103, (1994).

33 Butter, F., Scheibe, M., Morl, M. \& Mann, M. Unbiased RNA-protein interaction screen by quantitative proteomics. Proc Natl Acad Sci U S A 106, 10626-10631, (2009).

34 Scherrer, T., Mittal, N., Janga, S. C. \& Gerber, A. P. A screen for RNA-binding proteins in yeast indicates dual functions for many enzymes. PLoS One 5, e15499, (2010).

35 Tsvetanova, N. G., Klass, D. M., Salzman, J. \& Brown, P. O. Proteome-wide search reveals unexpected RNA-binding proteins in Saccharomyces cerevisiae. PLoS One 5, e12671., (2010).

36 Treiber, T., Treiber, N., Plessmann, U., Harlander, S., Daiss, J. L., Eichner, N., ... Meister, G. A Compendium of RNA-Binding Proteins that Regulate MicroRNA Biogenesis. Mol Cell 66, 270-284 e213, (2017).

37 Castello, A., Horos, R., Strein, C., Fischer, B., Eichelbaum, K., Steinmetz, L. M., ... . Hentze, M. W. System-wide identification of RNA-binding proteins by interactome capture. Nat Protoc 8, 491-500, (2013).

38 Baltz, A. G., Munschauer, M., Schwanhausser, B., Vasile, A., Murakawa, Y., Schueler, M., . . . Landthaler, M. The mRNA-Bound Proteome and Its Global Occupancy Profile on Protein-Coding Transcripts. Mol Cell 46, 674-690, (2012).

39 Castello, A., Fischer, B., Eichelbaum, K., Horos, R., Beckmann, B. M., Strein, C., . . . Hentze, M. W. Insights into RNA Biology from an Atlas of Mammalian mRNA-Binding Proteins. Cell 149, 1393-1406, (2012).

40 Castello, A., Fischer, B., Hentze, M. W. \& Preiss, T. RNA-binding proteins in Mendelian disease. Trends Genet 29, 318-327, (2013).

41 Strein, C., Alleaume, A. M., Rothbauer, U., Hentze, M. W. \& Castello, A. A versatile assay for RNA-binding proteins in living cells. RNA 20, 721-731, (2014).

42 Kramer, K., Sachsenberg, T., Beckmann, B. M., Qamar, S., Boon, K. L., Hentze, M. W., .. . Urlaub, H. Photo-cross-linking and high-resolution mass spectrometry for assignment of RNA-binding sites in RNA-binding proteins. Nat Methods 11, 10641070, (2014).

43 Beckmann, B. M., Horos, R., Fischer, B., Castello, A., Eichelbaum, K., Alleaume, A. M., . . . Hentze, M. W. The RNA-binding proteomes from yeast to man harbour conserved enigmRBPs. Nat Commun 6, 10127, (2015).

44 Castello, A., Fischer, B., Frese, C. K., Horos, R., Alleaume, A. M., Foehr, S., . . . Hentze, M. W. Comprehensive Identification of RNA-Binding Domains in Human Cells. Mol Cell 63, 696-710, (2016).

45 Kwon, S. C., Yi, H., Eichelbaum, K., Fohr, S., Fischer, B., You, K. T., ... Kim, V. N. The RNA-binding protein repertoire of embryonic stem cells. Nat Struct Mol Biol 20, 1122-1130, (2013).

46 Liao, Y., Castello, A., Fischer, B., Leicht, S., Foehr, S., Frese, C. K., . . Preiss, T. The Cardiomyocyte RNA-Binding Proteome: Links to Intermediary Metabolism and Heart Disease. Cell Rep 16, 1456-1469, (2016). 
Liepelt, A., Naarmann-de Vries, I. S., Simons, N., Eichelbaum, K., Foehr, S., Archer, S. K., . . . Ostareck-Lederer, A. Identification of RNA-binding proteins in macrophages by interactome capture. Mol Cell Proteomics 15, 2699-2674, (2016).

48 Mitchell, S. F., Jain, S., She, M. \& Parker, R. Global analysis of yeast mRNPs. Nat Struct Mol Biol 20, 127-133, (2013).

49 Matia-Gonzalez, A. M., Laing, E. E. \& Gerber, A. P. Conserved mRNA-binding proteomes in eukaryotic organisms. Nat Struct Mol Biol 22, 1027-1033, (2015).

50 Nandan, D., Thomas, S. A., Nguyen, A., Moon, K. M., Foster, L. J. \& Reiner, N. E. Comprehensive Identification of mRNA-Binding Proteins of Leishmania donovani by Interactome Capture. PLoS One 12, e0170068, (2017).

51 Bunnik, E. M., Batugedara, G., Saraf, A., Prudhomme, J., Florens, L. \& Le Roch, K. G. The mRNA-bound proteome of the human malaria parasite Plasmodium falciparum. Genome Biol 17, 147, (2016).

52 Lueong, S., Merce, C., Fischer, B., Hoheisel, J. D. \& Erben, E. D. Gene expression regulatory networks in Trypanosoma brucei: insights into the role of the mRNAbinding proteome. Mol Microbiol 100, 457-471, (2016).

53 Marondedze, C., Thomas, L., Serrano, N. L., Lilley, K. S. \& Gehring, C. The RNAbinding protein repertoire of Arabidopsis thaliana. Sci Rep 6, 29766, (2016).

54 Reichel, M., Liao, Y., Rettel, M., Ragan, C., Evers, M., Alleaume, A. M., . . . Millar, A. A. In Planta Determination of the mRNA-Binding Proteome of Arabidopsis Etiolated Seedlings. Plant Cell 28, 2435-2452, (2016).

55 Zhang, Z., Boonen, K., Ferrari, P., Schoofs, L., Janssens, E., van Noort, V., . . . Geuten, K. UV crosslinked mRNA-binding proteins captured from leaf mesophyll protoplasts. Plant Methods 12, 42, (2016).

56 Sysoev, V. O., Fischer, B., Frese, C. K., Gupta, I., Krijgsveld, J., Hentze, M. W., . . . Ephrussi, A. Global changes of the RNA-bound proteome during the maternal-tozygotic transition in Drosophila. Nat Commun 7, 12128, (2016).

57 Wessels, H. H., Imami, K., Baltz, A. G., Kolinski, M., Beldovskaya, A., Selbach, M., . . . Landthaler, M. The mRNA-bound proteome of the early fly embryo. Genome Res 26, 1000-1009, (2016).

58 Despic, V., Dejung, M., Gu, M., Krishnan, J., Zhang, J., Herzel, L., . . Neugebauer, K. M. Dynamic RNA-protein interactions underlie the zebrafish maternal-to-zygotic transition. Genome Res, (2017).

59 Sonnhammer, E. L. \& Ostlund, G. InParanoid 8: orthology analysis between 273 proteomes, mostly eukaryotic. Nucleic Acids Res 43, D234-239, (2015).

60 O'Brien, K. P., Remm, M. \& Sonnhammer, E. L. Inparanoid: a comprehensive database of eukaryotic orthologs. Nucleic Acids Res 33, D476-480, (2005).

61 Conrad, T., Albrecht, A. S., de Melo Costa, V. R., Sauer, S., Meierhofer, D. \& Orom, U. A. Serial interactome capture of the human cell nucleus. Nat Commun 7, 11212, (2016).

62 Gilbert, W. V., Bell, T. A. \& Schaening, C. Messenger RNA modifications: Form, distribution, and function. Science 352, 1408-1412, (2016).

63 Hoernes, T. P. \& Erlacher, M. D. Translating the epitranscriptome. Wiley Interdiscip Rev RNA 8, (2017).

64 Ciesla, J. Metabolic enzymes that bind RNA: yet another level of cellular regulatory network? Acta Biochim Pol 53, 11-32, (2006).

65 Hentze, M. W. \& Preiss, T. The REM phase of gene regulation. Trends Biochem Sci 35, 423-426, (2010). 
Castello, A., Hentze, M. W. \& Preiss, T. Metabolic Enzymes Enjoying New Partnerships as RNA-Binding Proteins. Trends Endocrinol Metab 26, 746-757, (2015).

67 Chu, E. \& Allegra, C. J. The role of thymidylate synthase as an RNA binding protein. Bioessays 18, 191-198, (1996).

68 Liu, J., Schmitz, J. C., Lin, X., Tai, N., Yan, W., Farrell, M., . . . Chu, E. Thymidylate synthase as a translational regulator of cellular gene expression. Biochim Biophys Acta 1587, 174-182, (2002).

69 Gerstberger, S., Hafner, M. \& Tuschl, T. A census of human RNA-binding proteins. Nat Rev Genet 15, 829-845, (2014).

70 ElAntak, L., Tzakos, A. G., Locker, N. \& Lukavsky, P. J. Structure of elF3b RNA recognition motif and its interaction with elF3j: structural insights into the recruitment of elF3b to the $40 \mathrm{~S}$ ribosomal subunit. J Biol Chem 282, 8165-8174, (2007).

71 Bono, F., Ebert, J., Lorentzen, E. \& Conti, E. The crystal structure of the exon junction complex reveals how it maintains a stable grip on mRNA. Cell 126, 713-725, (2006).

72 Klass, D. M., Scheibe, M., Butter, F., Hogan, G. J., Mann, M. \& Brown, P. O. Quantitative proteomic analysis reveals concurrent RNA-protein interactions and identifies new RNA-binding proteins in Saccharomyces cerevisiae. Genome Res 23, 1028-1038, (2013).

73 Brannan, K. W., Jin, W., Huelga, S. C., Banks, C. A., Gilmore, J. M., Florens, L., . . . Yeo, G. W. SONAR Discovers RNA-Binding Proteins from Analysis of Large-Scale Protein-Protein Interactomes. Mol Cell 64, 282-293, (2016).

74 Sundararaman, B., Zhan, L., Blue, S. M., Stanton, R., Elkins, K., Olson, S., . . . Yeo, G. W. Resources for the Comprehensive Discovery of Functional RNA Elements. Mol Cell 61, 903-913, (2016).

75 Huttlin, E. L., Ting, L., Bruckner, R. J., Gebreab, F., Gygi, M. P., Szpyt, J., . . Gygi, S. P. The BioPlex Network: A Systematic Exploration of the Human Interactome. Cell 162, 425-440, (2015).

76 Mukhopadhyay, R., Ray, P. S., Arif, A., Brady, A. K., Kinter, M. \& Fox, P. L. DAPKZIPK-L13a axis constitutes a negative-feedback module regulating inflammatory gene expression. Mol Cell 32, 371-382, (2008).

77 Arif, A., Jia, J., Mukhopadhyay, R., Willard, B., Kinter, M. \& Fox, P. L. Two-site phosphorylation of EPRS coordinates multimodal regulation of noncanonical translational control activity. Mol Cell 35, 164-180, (2009).

78 Arif, A., Jia, J., Moodt, R. A., DiCorleto, P. E. \& Fox, P. L. Phosphorylation of glutamyl-prolyl tRNA synthetase by cyclin-dependent kinase 5 dictates transcriptselective translational control. Proc Natl Acad Sci U S A 108, 1415-1420, (2011).

79 Clingman, C. C., Deveau, L. M., Hay, S. A., Genga, R. M., Shandilya, S. M., Massi, F. \& Ryder, S. P. Allosteric inhibition of a stem cell RNA-binding protein by an intermediary metabolite. eLife, e02848, (2014).

80 Haghighat, A. \& Sonenberg, N. elF4G dramatically enhances the binding of elF4E to the mRNA 5'-cap structure. J Biol Chem 272, 21677-21680, (1997).

81 Dabo, S. \& Meurs, E. F. dsRNA-dependent protein kinase PKR and its role in stress, signaling and HCV infection. Viruses 4, 2598-2635, (2012).

82 Habjan, M. \& Pichlmair, A. Cytoplasmic sensing of viral nucleic acids. Curr Opin Virol 11, 31-37, (2015).

83 Rehwinkel, J. RNA sensing: the more RIG-I the merrier? EMBO Rep 14, 751-752, (2013). 
Boucas, J., Fritz, C., Schmitt, A., Riabinska, A., Thelen, L., Peifer, M., . . Reinhardt, H. C. Label-Free Protein-RNA Interactome Analysis Identifies Khsrp Signaling Downstream of the p38/Mk2 Kinase Complex as a Critical Modulator of Cell Cycle Progression. PLoS One 10, e0125745, (2015).

85 Lasko, P. Posttranscriptional regulation in Drosophila oocytes and early embryos. Wiley Interdiscip Rev RNA 2, 408-416, (2011).

86 Altelaar, A. F., Frese, C. K., Preisinger, C., Hennrich, M. L., Schram, A. W., Timmers, H. T., . . . Mohammed, S. Benchmarking stable isotope labeling based quantitative proteomics. J Proteomics 88, 14-26, (2013).

87 Fernandez-Chamorro, J., Pineiro, D., Gordon, J. M., Ramajo, J., Francisco-Velilla, R., Macias, M. J. \& Martinez-Salas, E. Identification of novel non-canonical RNAbinding sites in Gemin5 involved in internal initiation of translation. Nucleic Acids Res 42, 5742-5754, (2014).

88 Schmidt, C., Kramer, K. \& Urlaub, H. Investigation of protein-RNA interactions by mass spectrometry--Techniques and applications. J Proteomics 75, 3478-3494, (2012).

89 Nagy, E. \& Rigby, W. F. Glyceraldehyde-3-phosphate dehydrogenase selectively binds AU-rich RNA in the NAD(+)-binding region (Rossmann fold). J Biol Chem 270, 2755-2763, (1995).

90 Chang, C. H., Curtis, J. D., Maggi, L. B., Jr., Faubert, B., Villarino, A. V., O'Sullivan, D., . . . Pearce, E. L. Posttranscriptional control of T cell effector function by aerobic glycolysis. Cell 153, 1239-1251, (2013).

91 Mullari, M., Lyon, D., Jensen, L. J. \& Nielsen, M. L. Specifying RNA-Binding Regions in Proteins by Peptide Cross-Linking and Affinity Purification. J Proteome Res 16, 2762-2772, (2017).

92 He, C., Sidoli, S., Warneford-Thomson, R., Tatomer, D. C., Wilusz, J. E., Garcia, B. A. \& Bonasio, R. High-Resolution Mapping of RNA-Binding Regions in the Nuclear Proteome of Embryonic Stem Cells. Mol Cell 64, 416-430, (2016).

93 Kaneko, S., Li, G., Son, J., Xu, C. F., Margueron, R., Neubert, T. A. \& Reinberg, D. Phosphorylation of the PRC2 component Ezh2 is cell cycle-regulated and upregulates its binding to ncRNA. Genes Dev 24, 2615-2620, (2010).

94 Kaneko, S., Son, J., Bonasio, R., Shen, S. S. \& Reinberg, D. Nascent RNA interaction keeps PRC2 activity poised and in check. Genes Dev 28, 1983-1988, (2014).

95 Kaneko, S., Son, J., Shen, S. S., Reinberg, D. \& Bonasio, R. PRC2 binds active promoters and contacts nascent RNAs in embryonic stem cells. Nat Struct Mol Biol 20, 1258-1264, (2013).

96 Ray, D., Kazan, H., Cook, K. B., Weirauch, M. T., Najafabadi, H. S., Li, X., . . . Hughes, T. R. A compendium of RNA-binding motifs for decoding gene regulation. Nature 499, 172-177, (2013).

97 Hafner, M., Landthaler, M., Burger, L., Khorshid, M., Hausser, J., Berninger, P., . . . Tuschl, T. Transcriptome-wide identification of RNA-binding protein and microRNA target sites by PAR-CLIP. Cell 141, 129-141, (2010).

98 König, J., Zarnack, K., Rot, G., Curk, T., Kayikci, M., Zupan, B., . . . Ule, J. iCLIP reveals the function of hnRNP particles in splicing at individual nucleotide resolution. Nat Struct Mol Biol 17, 909-915, (2010).

99 Van Nostrand, E. L., Pratt, G. A., Shishkin, A. A., Gelboin-Burkhart, C., Fang, M. Y., Sundararaman, B., . . . Yeo, G. W. Robust transcriptome-wide discovery of RNAbinding protein binding sites with enhanced CLIP (eCLIP). Nat Methods 13, 508-514, (2016). 
100 Lambert, N., Robertson, A., Jangi, M., McGeary, S., Sharp, P. A. \& Burge, C. B. RNA Bind-n-Seq: quantitative assessment of the sequence and structural binding specificity of RNA binding proteins. Mol Cell 54, 887-900, (2014).

101 Riley, K. J. \& Steitz, J. A. The "Observer Effect" in genome-wide surveys of proteinRNA interactions. Mol Cell 49, 601-604, (2013).

102 Steckelberg, A. L., Boehm, V., Gromadzka, A. M. \& Gehring, N. H. CWC22 connects pre-mRNA splicing and exon junction complex assembly. Cell Rep 2, 454-461, (2012).

103 Haberman, N., Huppertz, I., Attig, J., Konig, J., Wang, Z., Hauer, C., . . . Ule, J. Insights into the design and interpretation of iCLIP experiments. Genome Biol 18, 7, (2017).

104 Jarvelin, A. I., Noerenberg, M., Davis, I. \& Castello, A. The new (dis)order in RNA regulation. Cell Commun Signal 14, 9, (2016).

105 Basu, S. \& Bahadur, R. P. A structural perspective of RNA recognition by intrinsically disordered proteins. Cell Mol Life Sci 73, 4075-4084, (2016).

106 Thandapani, P., O'Connor, T. R., Bailey, T. L. \& Richard, S. Defining the RGG/RG motif. Mol Cell 50, 613-623, (2013).

107 Phan, A. T., Kuryavyi, V., Darnell, J. C., Serganov, A., Majumdar, A., llin, S., . . . Patel, D. J. Structure-function studies of FMRP RGG peptide recognition of an RNA duplex-quadruplex junction. Nat Struct Mol Biol 18, 796-804, (2011).

108 Han, T. W., Kato, M., Xie, S., Wu, L. C., Mirzaei, H., Pei, J., . . McKnight, S. L. Cellfree formation of RNA granules: bound RNAs identify features and components of cellular assemblies. Cell 149, 768-779, (2012).

109 Kato, M., Han, T. W., Xie, S., Shi, K., Du, X., Wu, L. C., . . McKnight, S. L. Cell-free formation of RNA granules: low complexity sequence domains form dynamic fibers within hydrogels. Cell 149, 753-767, (2012).

110 Vuzman, D., Azia, A. \& Levy, Y. Searching DNA via a "Monkey Bar" mechanism: the significance of disordered tails. $J$ Mol Biol 396, 674-684, (2010).

111 Casu, F., Duggan, B. M. \& Hennig, M. The arginine-rich RNA-binding motif of HIV-1 Rev is intrinsically disordered and folds upon RRE binding. Biophys $J$ 105, 10041017, (2013).

112 Gold, L., Janjic, N., Jarvis, T., Schneider, D., Walker, J. J., Wilcox, S. K. \& Zichi, D. Aptamers and the RNA world, past and present. Cold Spring Harb Perspect Biol 4, (2012).

113 Shi, H. \& Moore, P. B. The crystal structure of yeast phenylalanine tRNA at $1.93 \mathrm{~A}$ resolution: a classic structure revisited. RNA 6, 1091-1105, (2000).

114 de Breyne, S., Yu, Y., Unbehaun, A., Pestova, T. V. \& Hellen, C. U. Direct functional interaction of initiation factor elF4G with type 1 internal ribosomal entry sites. Proc Natl Acad Sci U S A 106, 9197-9202, (2009).

115 Sizova, D. V., Kolupaeva, V. G., Pestova, T. V., Shatsky, I. N. \& Hellen, C. U. Specific interaction of eukaryotic translation initiation factor 3 with the $5^{\prime}$ nontranslated regions of hepatitis $\mathrm{C}$ virus and classical swine fever virus RNAs. $J$ Virol 72, 4775-4782, (1998).

116 Quade, N., Boehringer, D., Leibundgut, M., van den Heuvel, J. \& Ban, N. Cryo-EM structure of Hepatitis $C$ virus IRES bound to the human ribosome at 3.9-A resolution. Nat Commun 6, 7646, (2015).

117 Clemson, C. M., Hutchinson, J. N., Sara, S. A., Ensminger, A. W., Fox, A. H., Chess, A. \& Lawrence, J. B. An architectural role for a nuclear noncoding RNA: NEAT1 RNA is essential for the structure of paraspeckles. Mol Cell 33, 717-726, (2009). 
118 Chu, E., Koeller, D. M., Casey, J. L., Drake, J. C., Chabner, B. A., Elwood, P. C., ... Allegra, C. J. Autoregulation of human thymidylate synthase messenger RNA translation by thymidylate synthase. Proc Natl Acad Sci U S A 88, 8977-8981, (1991).

119 Muckenthaler, M. U., Rivella, S., Hentze, M. W. \& Galy, B. A Red Carpet for Iron Metabolism. Cell 168, 344-361, (2017).

120 Walden, W. E., Selezneva, A. I., Dupuy, J., Volbeda, A., Fontecilla-Camps, J. C., Theil, E. C. \& Volz, K. Structure of dual function iron regulatory protein 1 complexed with ferritin IRE-RNA. Science 314, 1903-1908, (2006).

121 Tristan, C., Shahani, N., Sedlak, T. W. \& Sawa, A. The diverse functions of GAPDH: views from different subcellular compartments. Cell Signal 23, 317-323, (2011).

122 Rodriguez-Pascual, F., Redondo-Horcajo, M., Magan-Marchal, N., Lagares, D., Martinez-Ruiz, A., Kleinert, H. \& Lamas, S. Glyceraldehyde-3-phosphate dehydrogenase regulates endothelin-1 expression by a novel, redox-sensitive mechanism involving mRNA stability. Mol Cell Biol 28, 7139-7155, (2008).

123 Xing, S. \& Poirier, Y. The protein acetylome and the regulation of metabolism. Trends Plant Sci 17, 423-430, (2012).

124 Arif, W., Datar, G. \& Kalsotra, A. Intersections of post-transcriptional gene regulatory mechanisms with intermediary metabolism. Biochim Biophys Acta 1860, 349-362, (2017).

125 Campbell, Z. T. \& Wickens, M. Probing RNA-protein networks: biochemistry meets genomics. Trends Biochem Sci 40, 157-164, (2015).

126 König, J., Zarnack, K., Luscombe, N. M. \& Ule, J. Protein-RNA interactions: new genomic technologies and perspectives. Nat Rev Genet 13, 77-83, (2011).

127 Nostrand, E. L., Van, F., P., Pratt, G. A., Wang, X., Wei, X., Blue, S. M. \& Yeo, G. W. A Large-Scale Binding and Functional Map of Human RNA Binding Proteins. bioRxiv 179648, (2017).

128 Papasaikas, P., Tejedor, J. R., Vigevani, L. \& Valcarcel, J. Functional splicing network reveals extensive regulatory potential of the core spliceosomal machinery. Mol Cell 57, 7-22, (2015).

129 Tejedor, J. R., Papasaikas, P. \& Valcarcel, J. Genome-wide identification of Fas/CD95 alternative splicing regulators reveals links with iron homeostasis. $\mathrm{Mol}$ Cell 57, 23-38, (2015).

130 Choudhury, N. R., Nowak, J. S., Zuo, J., Rappsilber, J., Spoel, S. H. \& Michlewski, G. Trim25 Is an RNA-Specific Activator of Lin28a/TuT4-Mediated Uridylation. Cell Rep 9, 1265-1272, (2014).

131 Manokaran, G., Finol, E., Wang, C., Gunaratne, J., Bahl, J., Ong, E. Z., ... Ooi, E. E. Dengue subgenomic RNA binds TRIM25 to inhibit interferon expression for epidemiological fitness. Science 350, 217-221, (2015).

132 Gebhardt, A., Habjan, M., Benda, C., Meiler, A., Haas, D. A., Hein, M. Y., . . . Pichlmair, A. mRNA export through an additional cap-binding complex consisting of NCBP1 and NCBP3. Nat Commun 6, 8192, (2015).

133 Popow, J., Alleaume, A. M., Curk, T., Schwarzl, T., Sauer, S. \& Hentze, M. W. FASTKD2 is an RNA-binding protein required for mitochondrial RNA processing and translation. RNA 21, 1873-1884, (2015).

134 Gaspar, I., Sysoev, V., Komissarov, A. \& Ephrussi, A. An RNA-binding atypical tropomyosin recruits kinesin-1 dynamically to oskar mRNPs. EMBO J 36, 319-333, (2017). 
135 Yang, S. Y., He, X. Y., Isaacs, C., Dobkin, C., Miller, D. \& Philipp, M. Roles of 17beta-hydroxysteroid dehydrogenase type 10 in neurodegenerative disorders. $J$ Steroid Biochem Mol Biol 143, 460-472, (2014).

136 Rauschenberger, K., Scholer, K., Sass, J. O., Sauer, S., Djuric, Z., Rumig, C., ... . Zschocke, J. A non-enzymatic function of 17 beta-hydroxysteroid dehydrogenase type 10 is required for mitochondrial integrity and cell survival. EMBO Mol Med 2, 5162, (2010).

137 Holzmann, J., Frank, P., Loffler, E., Bennett, K. L., Gerner, C. \& Rossmanith, W. RNase $P$ without RNA: identification and functional reconstitution of the human mitochondrial tRNA processing enzyme. Cell 135, 462-474, (2008).

138 Vilardo, E. \& Rossmanith, W. Molecular insights into HSD10 disease: impact of SDR5C1 mutations on the human mitochondrial RNase P complex. Nucleic Acids Res 43, 6649, (2015).

139 Antonicka, H. \& Shoubridge, E. A. Mitochondrial RNA Granules Are Centers for Posttranscriptional RNA Processing and Ribosome Biogenesis. Cell Rep, (2015).

140 Ghezzi, D., Saada, A., D'Adamo, P., Fernandez-Vizarra, E., Gasparini, P., Tiranti, V., Zeviani, M. FASTKD2 nonsense mutation in an infantile mitochondrial encephalomyopathy associated with cytochrome c oxidase deficiency. Am J Hum Genet 83, 415-423, (2008).

141 Nagata, T., Suzuki, S., Endo, R., Shirouzu, M., Terada, T., Inoue, M., . . Yokoyama, $\mathrm{S}$. The RRM domain of poly(A)-specific ribonuclease has a noncanonical binding site for mRNA cap analog recognition. Nucleic Acids Res 36, 4754-4767, (2008).

142 Faehnle, C. R., Walleshauser, J. \& Joshua-Tor, L. Mechanism of Dis312 substrate recognition in the Lin28-let-7 pathway. Nature 514, 252-256, (2014).

143 Gack, M. U., Shin, Y. C., Joo, C. H., Urano, T., Liang, C., Sun, L., . . . Jung, J. U. TRIM25 RING-finger E3 ubiquitin ligase is essential for RIG-I-mediated antiviral activity. Nature 446, 916-920, (2007).

144 Chapman, E. G., Costantino, D. A., Rabe, J. L., Moon, S. L., Wilusz, J., Nix, J. C. \& Kieft, J. S. The structural basis of pathogenic subgenomic flavivirus RNA (sfRNA) production. Science 344, 307-310, (2014).

145 Kanakkanthara, A., Jeganathan, K. B., Limzerwala, J. F., Baker, D. J., Hamada, M., Nam, H. J., ... van Deursen, J. M. Cyclin A2 is an RNA binding protein that controls Mre11 mRNA translation. Science 353, 1549-1552, (2016).

146 Pashev, I. G., Dimitrov, S. I. \& Angelov, D. Crosslinking proteins to nucleic acids by ultraviolet laser irradiation. Trends Biochem Sci 16, 323-326, (1991).

147 Van Nostrand, E. L., Gelboin-Burkhart, C., Wang, R., Pratt, G. A., Blue, S. M. \& Yeo, G. W. CRISPR/Cas9-mediated integration enables TAG-eCLIP of endogenously tagged RNA binding proteins. Methods 118, 50-59, (2016).

148 Wang, X., Zhao, B. S., Roundtree, I. A., Lu, Z., Han, D., Ma, H., . . . He, C. N(6)methyladenosine Modulates Messenger RNA Translation Efficiency. Cell 161, 13881399, (2015).

149 Zhou, J., Wan, J., Gao, X., Zhang, X., Jaffrey, S. R. \& Qian, S. B. Dynamic m(6)A mRNA methylation directs translational control of heat shock response. Nature 526, 591-594, (2015).

150 Wang, X., Lu, Z., Gomez, A., Hon, G. C., Yue, Y., Han, D., . . . He, C. N6methyladenosine-dependent regulation of messenger RNA stability. Nature 505, 117-120, (2014).

151 Wang, Y., Li, Y., Toth, J. I., Petroski, M. D., Zhang, Z. \& Zhao, J. C. N6methyladenosine modification destabilizes developmental regulators in embryonic stem cells. Nat Cell Biol 16, 191-198, (2014). 
152 Batista, P. J., Molinie, B., Wang, J., Qu, K., Zhang, J., Li, L., . . Chang, H. Y. m(6)A RNA modification controls cell fate transition in mammalian embryonic stem cells. Cell Stem Cell 15, 707-719, (2014).

153 Geula, S., Moshitch-Moshkovitz, S., Dominissini, D., Mansour, A. A., Kol, N., Salmon-Divon, M., . . . Hanna, J. H. Stem cells. m6A mRNA methylation facilitates resolution of naive pluripotency toward differentiation. Science 347, 1002-1006, (2015).

154 Zhao, B. S., Wang, X., Beadell, A. V., Lu, Z., Shi, H., Kuuspalu, A., ... He, C. m6Adependent maternal mRNA clearance facilitates zebrafish maternal-to-zygotic transition. Nature 542, 475-478, (2017).

155 Liu, N., Dai, Q., Zheng, G., He, C., Parisien, M. \& Pan, T. N(6)-methyladenosinedependent RNA structural switches regulate RNA-protein interactions. Nature 518, 560-564, (2015).

156 Lex, A., Gehlenborg, N., Strobelt, H., Vuillemot, R. \& Pfister, H. UpSet: Visualization of Intersecting Sets. IEEE Trans Vis Comput Graph 20, 1983-1992, (2014).

157 Altelaar, A. F., Munoz, J. \& Heck, A. J. Next-generation proteomics: towards an integrative view of proteome dynamics. Nat Rev Genet 14, 35-48, (2013).

158 Prevot, D., Darlix, J. L. \& Ohlmann, T. Conducting the initiation of protein synthesis: the role of elF4G. Biol Cell 95, 141-156, (2003).

159 Balachandran, S. \& Barber, G. N. PKR in innate immunity, cancer, and viral oncolysis. Methods Mol Biol 383, 277-301, (2007). 


\section{SUPPLEMENTARY INFORMATION}

Supplementary Figure 1. Comparison of RNA interactome capture data with in silico methods to identify RNA-binding proteins (RBPs). Venn diagrams showing overlaps of the human RNA interactome datasets with a curated list of RBPs ${ }^{1}$ (a) or with SONAR-identified RBPs ${ }^{2}$ (b). (c) Venn diagram showing the overlap between the Saccharomyces cerevisiae RNA interactome superset ${ }^{3-5}$ with the protein array-based, in vitro approach to identify $\operatorname{RBPs}^{6,7}$ and SONAR-identified RBPs in yeast ${ }^{2}$. (d) Functional annotation of the mouse, human and yeast RBP supersets (all identified RBPs in mouse $(\mathrm{Mm})^{8-12}$, human $(\mathrm{H})^{4,13-17}$ and yeast $(\mathrm{Sc})$ studies) ${ }^{3-7,15}$. Domain classifications ${ }^{2,14}$ were combined to annotate proteins with known RNA-binding domains. Current versions of GO terms include the RBPs of early interactomes ${ }^{13,14}$.

Supplementary Table 1. Curated table of RBP supersets identified in Homo sapiens ${ }^{4,13-17}$, Mus musculus) ${ }^{8,9,11,12}$, Saccharomyces cerevisiae ${ }^{3-5}$, Drosophila melanogaster $^{18,19}$, Arabidopsis thaliana ${ }^{20-22}$ and Caenorhabditis elegans ${ }^{5}$ using RNA interactome capture. RNA-binding proteomes generated with RNA interactome capture are compiled in Table S1. Due to ambiguous or outdated identifier (ID) annotations, our reported protein numbers, which are based on updated annotations and curation, occasionally differ from those in the original publications. We used the most stringent cutoffs provided by the original publications. Also note that the current gene ontology (GO) term "RNA binding" already includes the initial RNA interactome studies ${ }^{13,14}$. For human, mouse and yeast, 'Metabolism' has been annotated as 'true', when the respective RBP was listed under 'metabolism' in 'Reactome' (version 60). The 'Enzyme' annotation is 'true' when the protein is listed in in the six enzyme commission groups (EC 1-6) from the IntEnz database (release May 2017). "Metabolic enzyme" is "true" when the RBP fulfils our definition of metabolic enzyme. 'Metabolic enzyme' is an ambiguous term to define, and different authors have applied different criteria. For the purpose of this review, we generated a working list by mapping RBPs to "Metabolism" in the Reactome database (version 60) ${ }^{23}$. The resulting proteins were manually curated by removing genes whose protein products are not classified in the six enzyme commission groups (EC 1-6) from the IntEnz database (release May 2017) ${ }^{24}$, but retaining the subunits of ATP synthase and the respiratory chain complexes on the list of metabolic enzymes.

\section{REFERENCES}


1 Gerstberger, S., Hafner, M. \& Tuschl, T. A census of human RNAbinding proteins. Nat Rev Genet 15, 829-845, (2014).

2 Brannan, K. W., Jin, W., Huelga, S. C., Banks, C. A., Gilmore, J. M., Florens, L., ... Yeo, G. W. SONAR Discovers RNA-Binding Proteins from Analysis of Large-Scale Protein-Protein Interactomes. Mol Cell 64, 282-293, (2016).

3 Mitchell, S. F., Jain, S., She, M. \& Parker, R. Global analysis of yeast mRNPs. Nat Struct Mol Biol 20, 127-133, (2013).

4 Beckmann, B. M., Horos, R., Fischer, B., Castello, A., Eichelbaum, K., Alleaume, A. M., ... Hentze, M. W. The RNA-binding proteomes from yeast to man harbour conserved enigmRBPs. Nat Commun 6, 10127, (2015).

5 Matia-Gonzalez, A. M., Laing, E. E. \& Gerber, A. P. Conserved mRNAbinding proteomes in eukaryotic organisms. Nat Struct Mol Biol 22, 10271033, (2015).

6 Scherrer, T., Mittal, N., Janga, S. C. \& Gerber, A. P. A screen for RNAbinding proteins in yeast indicates dual functions for many enzymes. PLoS One 5, e15499, (2010).

7 Tsvetanova, N. G., Klass, D. M., Salzman, J. \& Brown, P. O. Proteomewide search reveals unexpected RNA-binding proteins in

Saccharomyces cerevisiae. PLoS One 5, e12671., (2010).

Kwon, S. C., Yi, H., Eichelbaum, K., Fohr, S., Fischer, B., You, K. T., . . . Kim, V. N. The RNA-binding protein repertoire of embryonic stem cells. Nat Struct Mol Biol 20, 1122-1130, (2013).

9 Boucas, J., Fritz, C., Schmitt, A., Riabinska, A., Thelen, L., Peifer, M., . . . Reinhardt, H. C. Label-Free Protein-RNA Interactome Analysis Identifies Khsrp Signaling Downstream of the p38/Mk2 Kinase Complex as a Critical Modulator of Cell Cycle Progression. PLoS One 10, e0125745, (2015).

10 He, C., Sidoli, S., Warneford-Thomson, R., Tatomer, D. C., Wilusz, J. E., Garcia, B. A. \& Bonasio, R. High-Resolution Mapping of RNA-Binding Regions in the Nuclear Proteome of Embryonic Stem Cells. Mol Cell 64, 416-430, (2016).

11 Liao, Y., Castello, A., Fischer, B., Leicht, S., Foehr, S., Frese, C. K., . . Preiss, T. The Cardiomyocyte RNA-Binding Proteome: Links to Intermediary Metabolism and Heart Disease. Cell Rep 16, 1456-1469, (2016).

12 Liepelt, A., Naarmann-de Vries, I. S., Simons, N., Eichelbaum, K., Foehr, S., Archer, S. K., . . . Ostareck-Lederer, A. Identification of RNA-binding proteins in macrophages by interactome capture. Mol Cell Proteomics 15, 2699-2674, (2016).

13 Baltz, A. G., Munschauer, M., Schwanhausser, B., Vasile, A., Murakawa, Y., Schueler, M., ... Landthaler, M. The mRNA-Bound Proteome and Its Global Occupancy Profile on Protein-Coding Transcripts. Mol Cell 46, 674-690, (2012).

14 Castello, A., Fischer, B., Eichelbaum, K., Horos, R., Beckmann, B. M., Strein, C., .. . Hentze, M. W. Insights into RNA Biology from an Atlas of Mammalian mRNA-Binding Proteins. Cell 149, 1393-1406, (2012). Kramer, K., Sachsenberg, T., Beckmann, B. M., Qamar, S., Boon, K. L., Hentze, M. W., . . . Urlaub, H. Photo-cross-linking and high-resolution 
mass spectrometry for assignment of RNA-binding sites in RNA-binding proteins. Nat Methods 11, 1064-1070, (2014).

16 Castello, A., Fischer, B., Frese, C. K., Horos, R., Alleaume, A. M., Foehr, S., ... Hentze, M. W. Comprehensive Identification of RNA-Binding Domains in Human Cells. Mol Cell 63, 696-710, (2016).

17 Conrad, T., Albrecht, A. S., de Melo Costa, V. R., Sauer, S., Meierhofer, D. \& Orom, U. A. Serial interactome capture of the human cell nucleus. Nat Commun 7, 11212, (2016).

18 Sysoev, V. O., Fischer, B., Frese, C. K., Gupta, I., Krijgsveld, J., Hentze, M. W., . . Ephrussi, A. Global changes of the RNA-bound proteome during the maternal-to-zygotic transition in Drosophila. Nat Commun 7, 12128, (2016).

19 Wessels, H. H., Imami, K., Baltz, A. G., Kolinski, M., Beldovskaya, A., Selbach, M., ... Landthaler, M. The mRNA-bound proteome of the early fly embryo. Genome Res 26, 1000-1009, (2016).

20 Marondedze, C., Thomas, L., Serrano, N. L., Lilley, K. S. \& Gehring, C. The RNA-binding protein repertoire of Arabidopsis thaliana. Sci Rep 6, 29766, (2016).

21 Reichel, M., Liao, Y., Rettel, M., Ragan, C., Evers, M., Alleaume, A. M., . . . Millar, A. A. In Planta Determination of the mRNA-Binding Proteome of Arabidopsis Etiolated Seedlings. Plant Cell 28, 2435-2452, (2016).

22 Zhang, Z., Boonen, K., Ferrari, P., Schoofs, L., Janssens, E., van Noort, V., . . . Geuten, K. UV crosslinked mRNA-binding proteins captured from leaf mesophyll protoplasts. Plant Methods 12, 42, (2016).

23 Croft, D., Mundo, A. F., Haw, R., Milacic, M., Weiser, J., Wu, G., . . . D'Eustachio, P. The Reactome pathway knowledgebase. Nucleic Acids Res 42, D472-477, (2014).

24 Fleischmann, A., Darsow, M., Degtyarenko, K., Fleischmann, W., Boyce, S., Axelsen, K. B., . . . Apweiler, R. IntEnz, the integrated relational enzyme database. Nucleic Acids Res 32, D434-437, (2004). 
a RBP acting on RNA

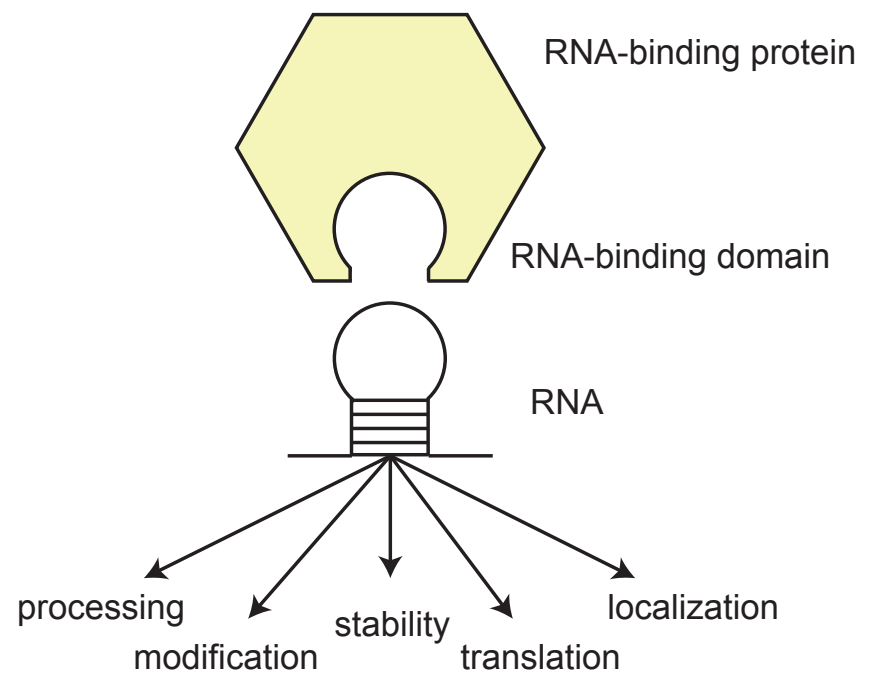

b RNA acting on RBP

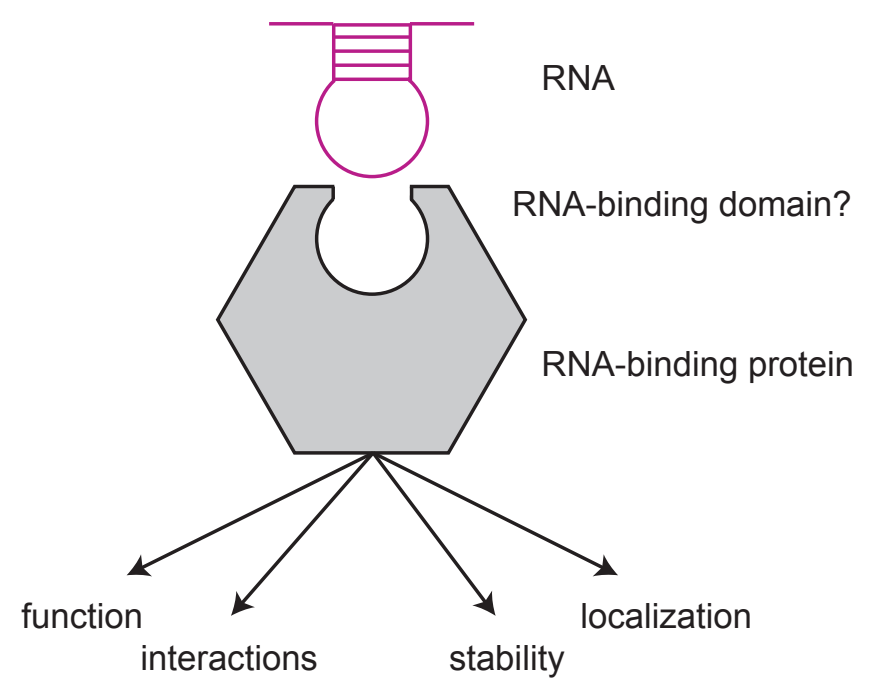

Figure 1 
a Identified RNA-binding proteins

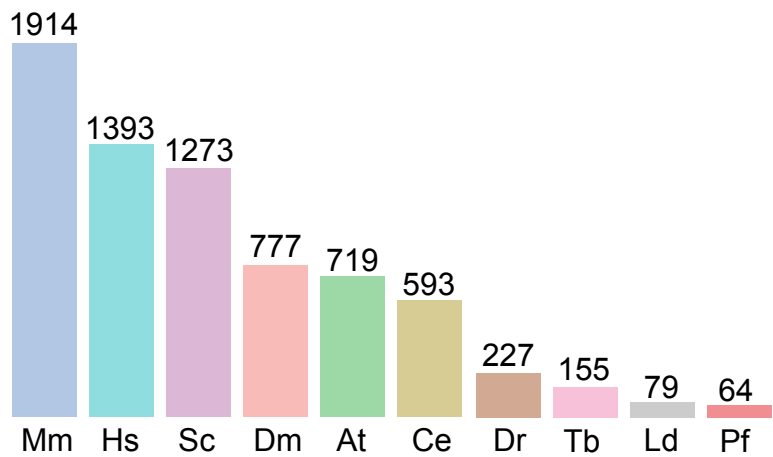
(6) (6)
(6) (8)
(2)
(3) (1)

e Drosophila melanogaster RBPs

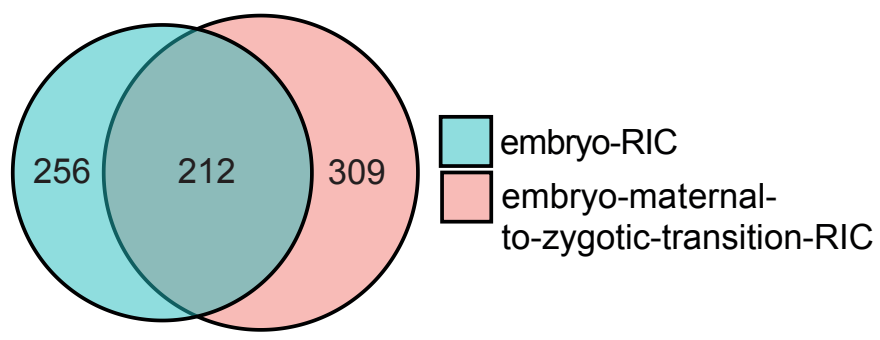

b Homo sapiens RBPs
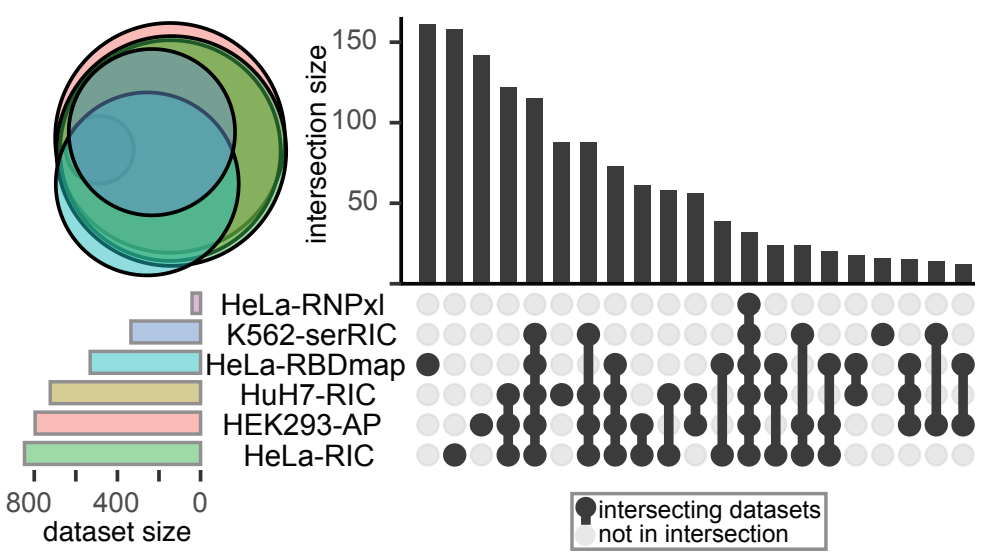

C Mus musculus RBPs
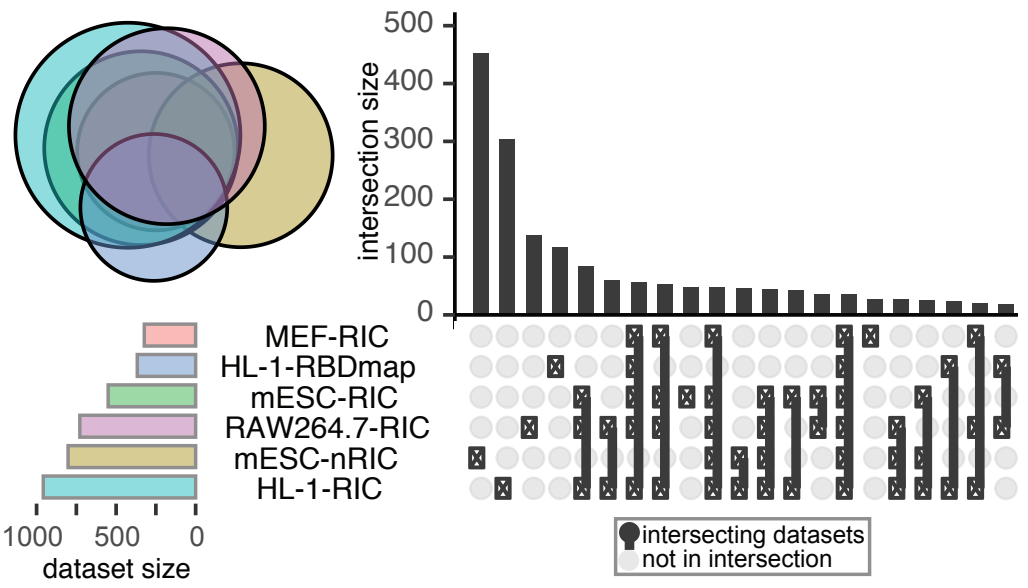

f Arabidopsis thaliana RBPs

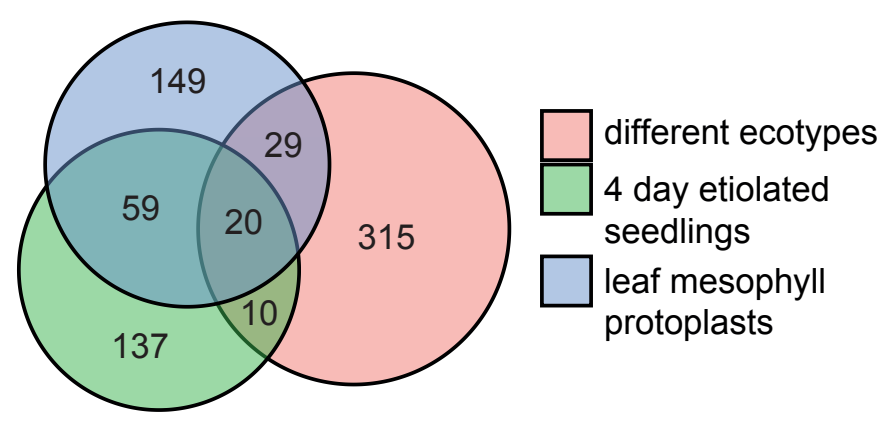

d Saccharomyces cerevisiae RBPs

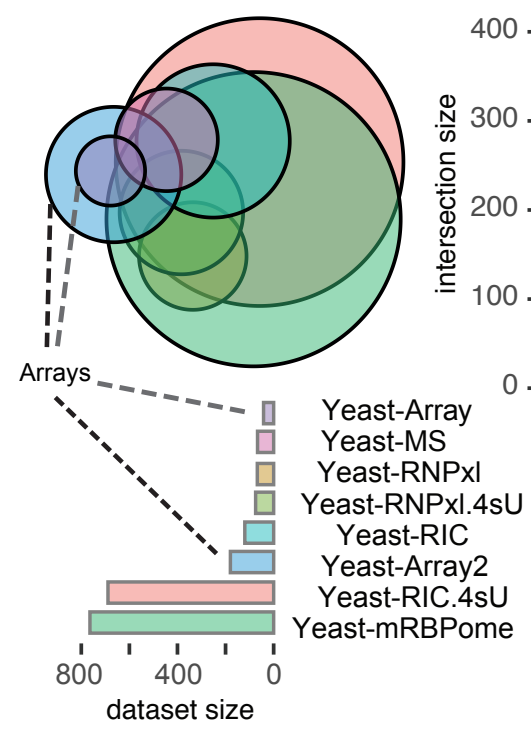

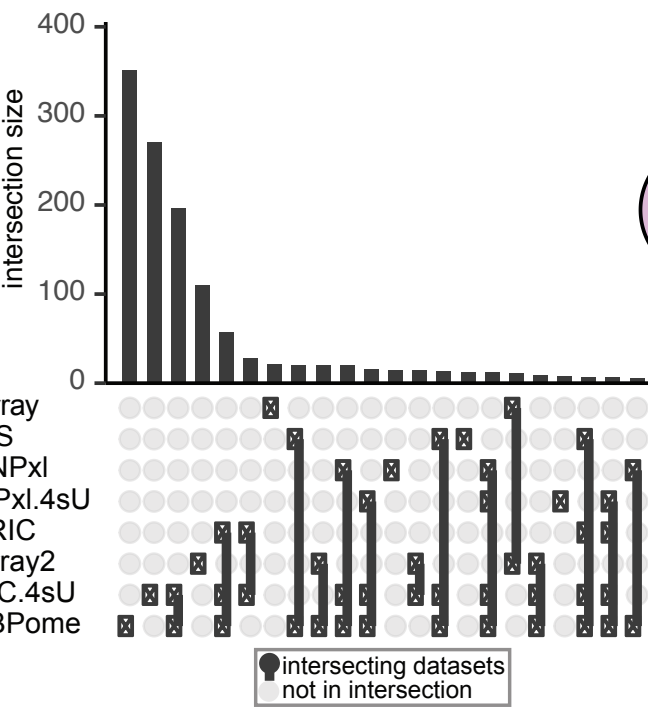

h Human orthologues

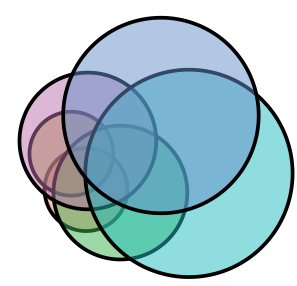

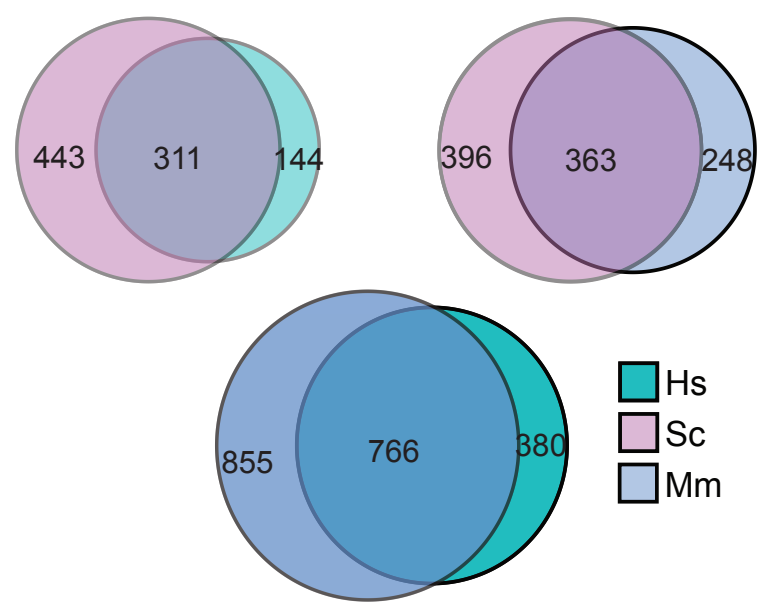

g InParanoid 6 cluster comparison

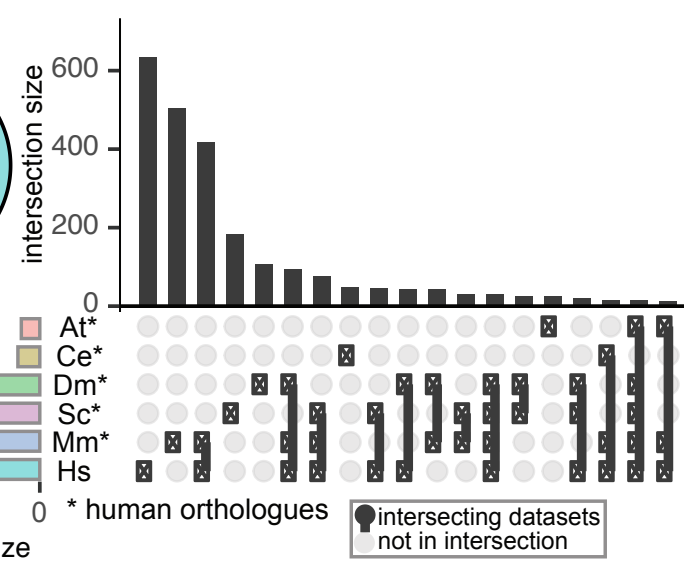

Figure 2 
a

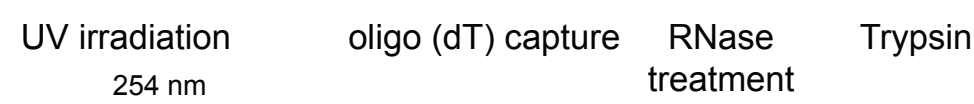

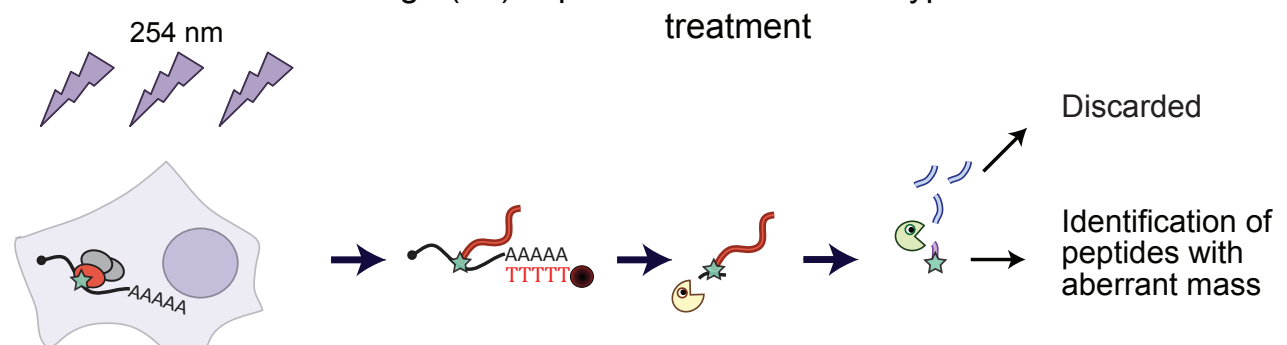

b

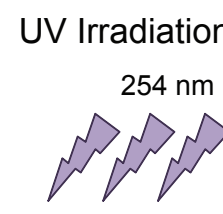

Oligo(dT) ArgC or LysC

Oligo(dT)

capture

RNase and

capture digestion

supernatant trypsin

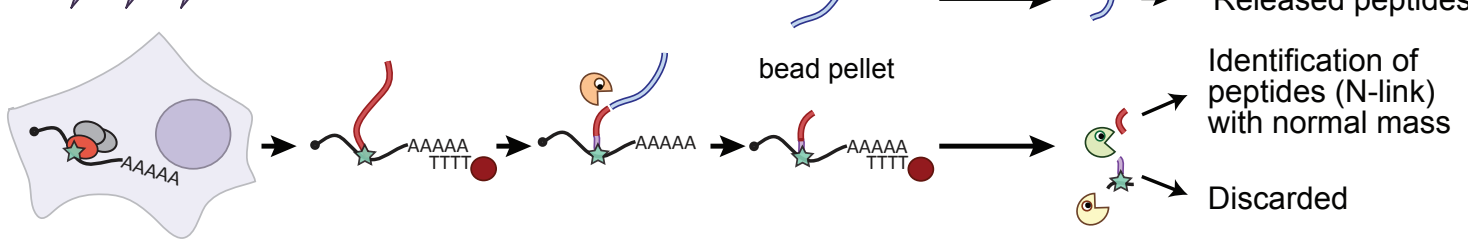

C UV Irradiation

RNase and trypsin

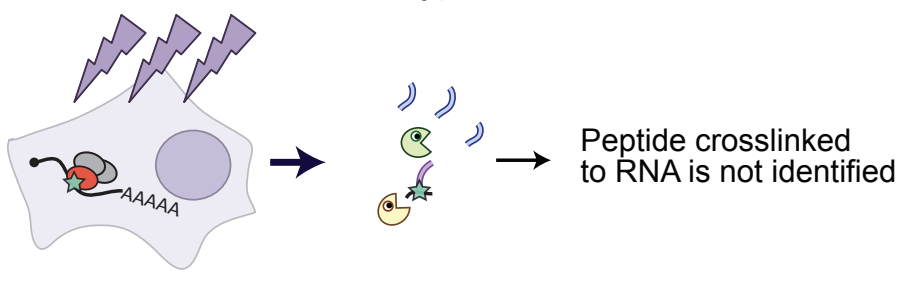

non irradiated

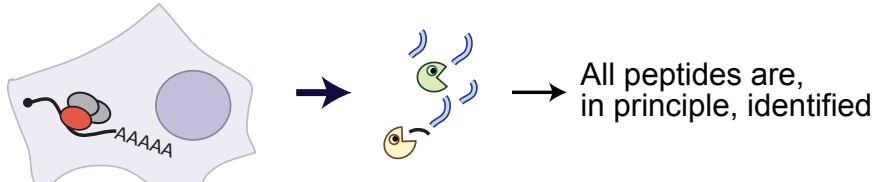

oligo(dT) beads
RNase
trypsin
amino acid - nucleotide
crosslink
RBP
denatured RBP
RBP peptide
croshboring peptide


a Classical mode of RNA-binding: high specificity
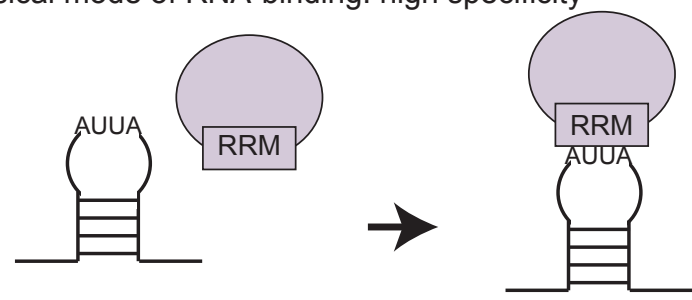

Effect on RNA

$\rightarrow$ processing, location, stability, translation

b Low target specificity RNA-binding
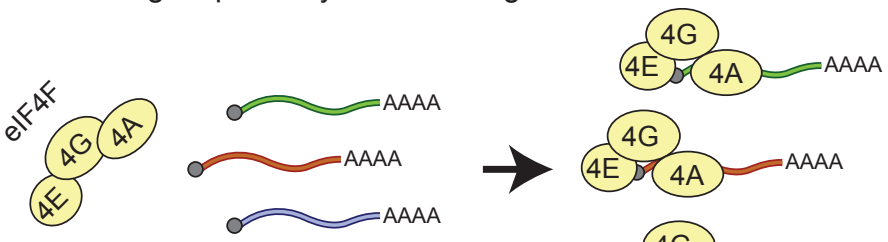

4G

4 E 4 A

$$
\text { (4E) } 4 \mathrm{~A}=\mathrm{A}=\mathrm{AAA}
$$

C Deposition by protein-protein interactions
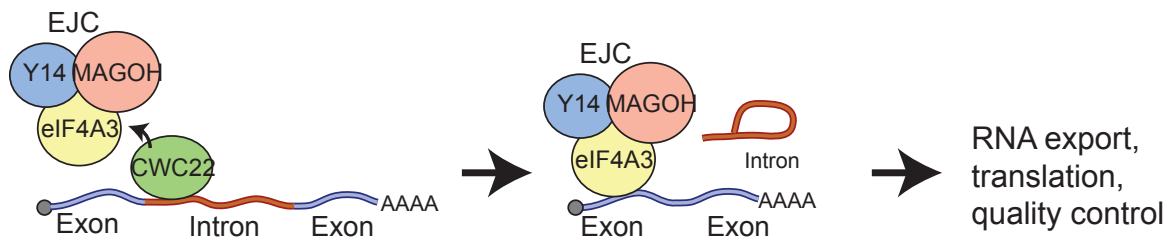

d RNA binding by protein disordered regions: co-folding
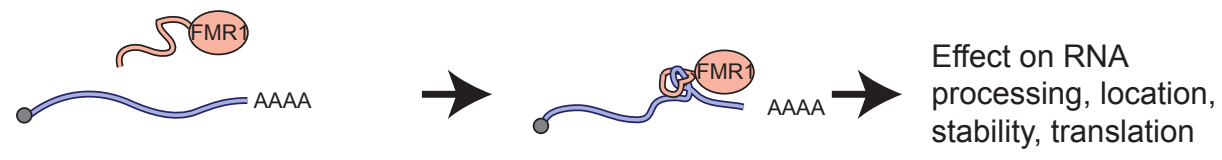

e Interactions by shape-complementarity
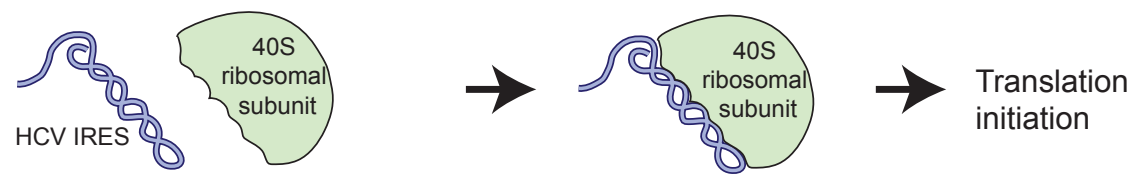

f Complex scaffold

g Modulation of protein's activity
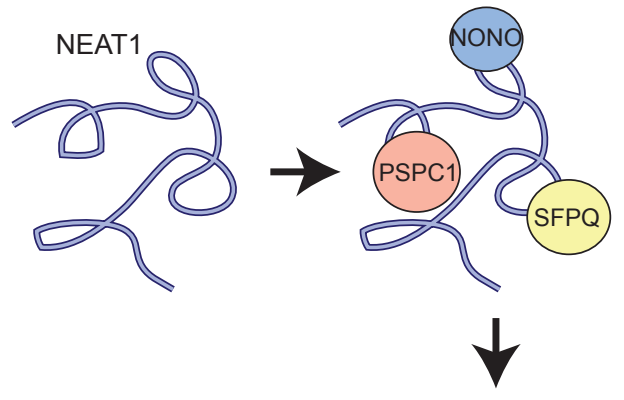

Protein storage

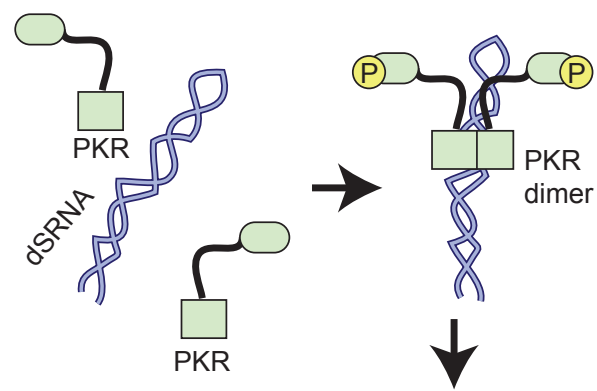

Shut off protein synthesis by elF2a phosphorylation

h Moonlighting RNA binding activity: regulation by metabolites

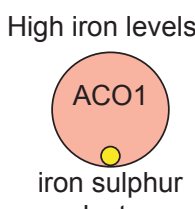

Low iron levels

$$
\text { cluster }
$$
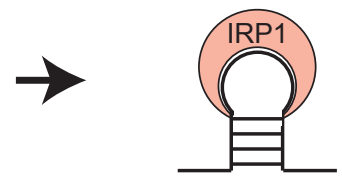

RNA stability

translation

\section{Figure 4}


a
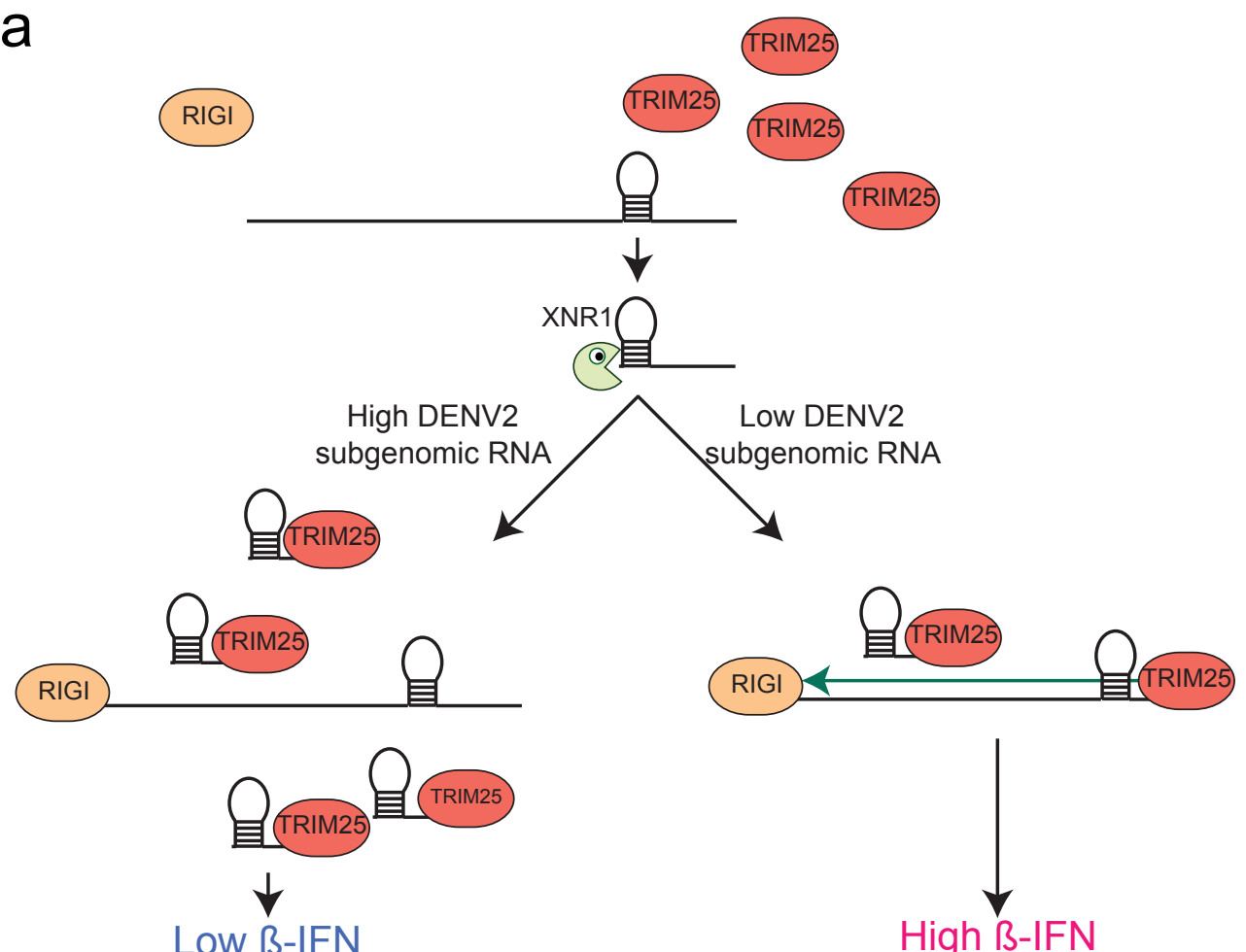

b

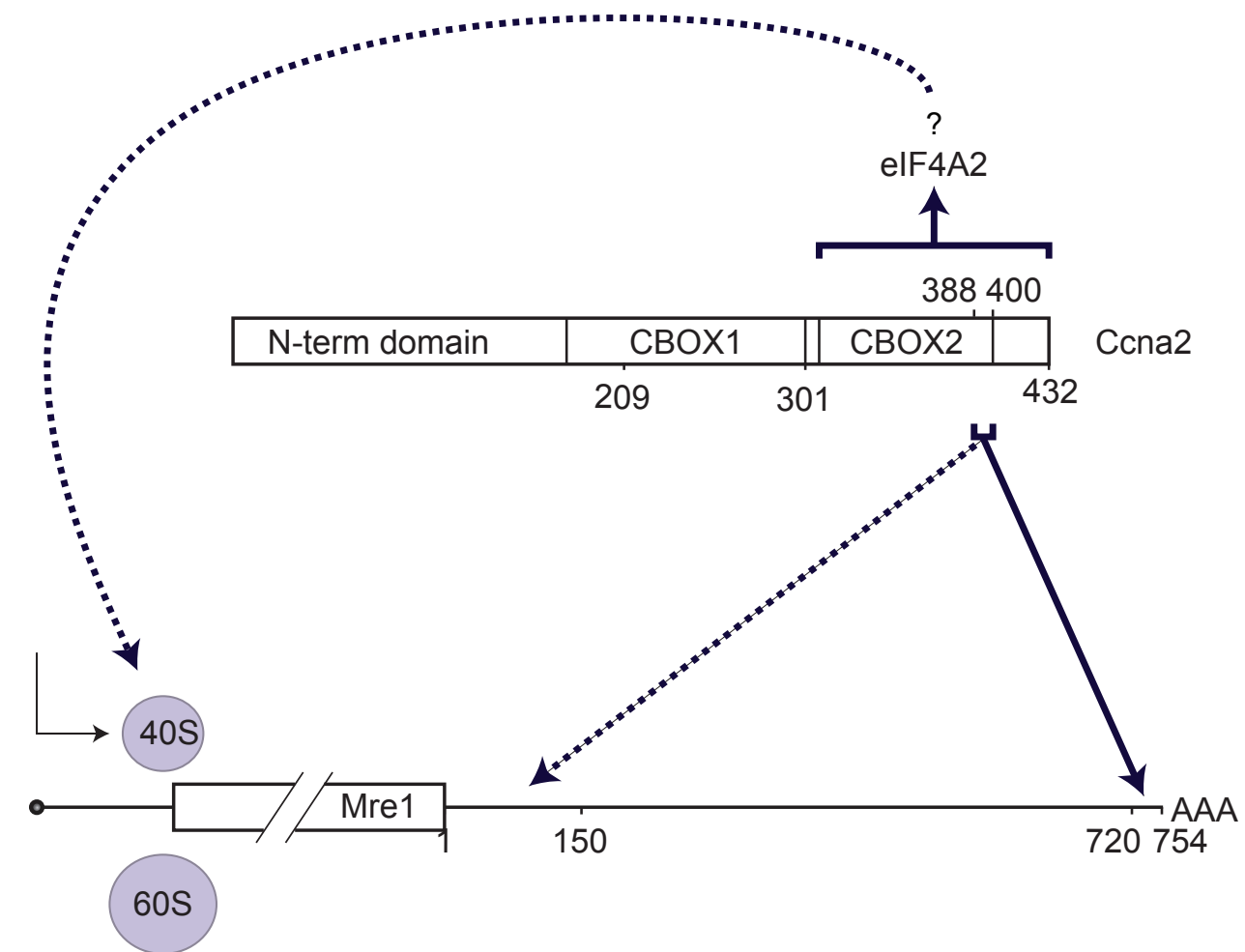

Figure 5 
a Immobilised

Incubation with

Quantitative

RNA probe

cellular extracts

proteomics
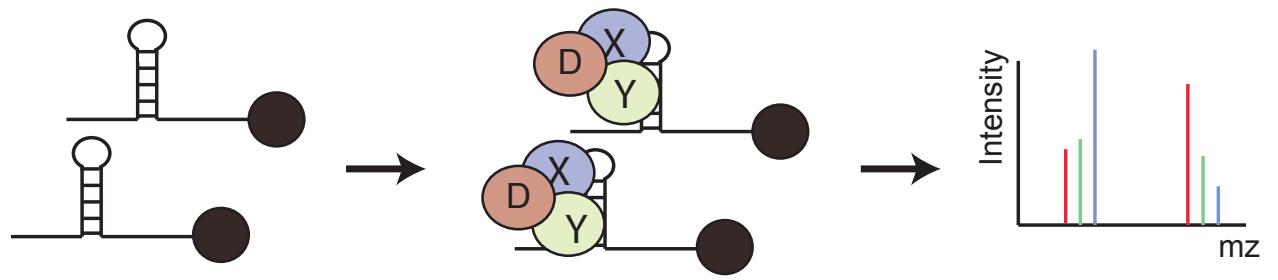

b Protein array

Incubation with

Fluorescence measurement fluorescently-labelled RNA at each protein spot
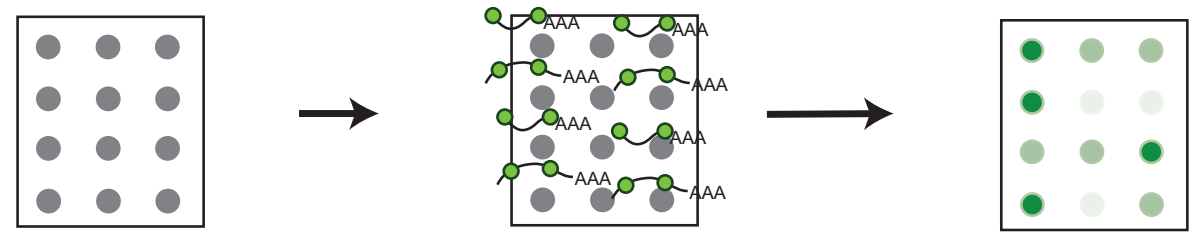

C Labelling

UV irradiation oligo ( $\mathrm{dT}$ ) capture RNase Quantitative treatment proteomics

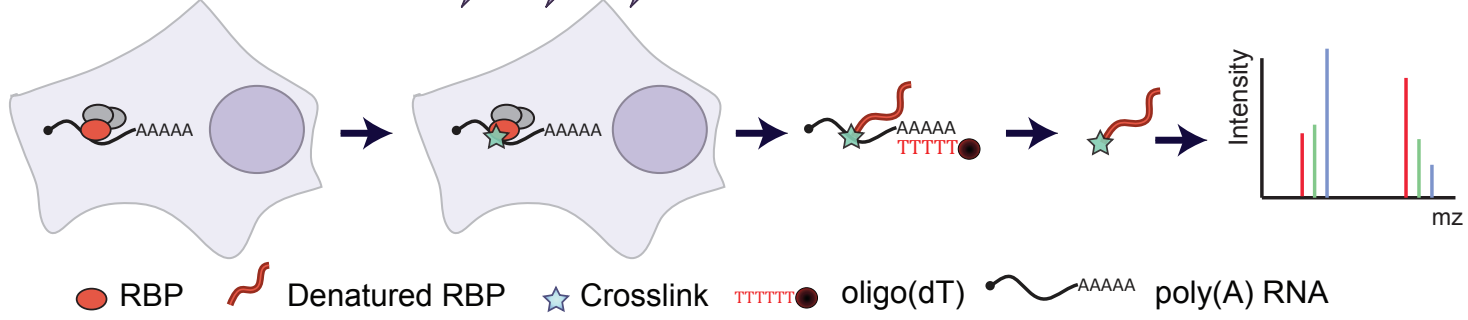

d UV irradiation $\begin{gathered}\text { Iysis and } \\ \text { RNase } \\ \text { treatment }\end{gathered}$ IP and 3' $\begin{gathered}\text { Complex size } \\ \text { adaptor ligation }\end{gathered} \begin{gathered}\text { Reverse } \\ \text { selection and } \\ \text { proteinase } K \\ \text { treatment }\end{gathered} \rightarrow$ 
a Functional analysis of supersets

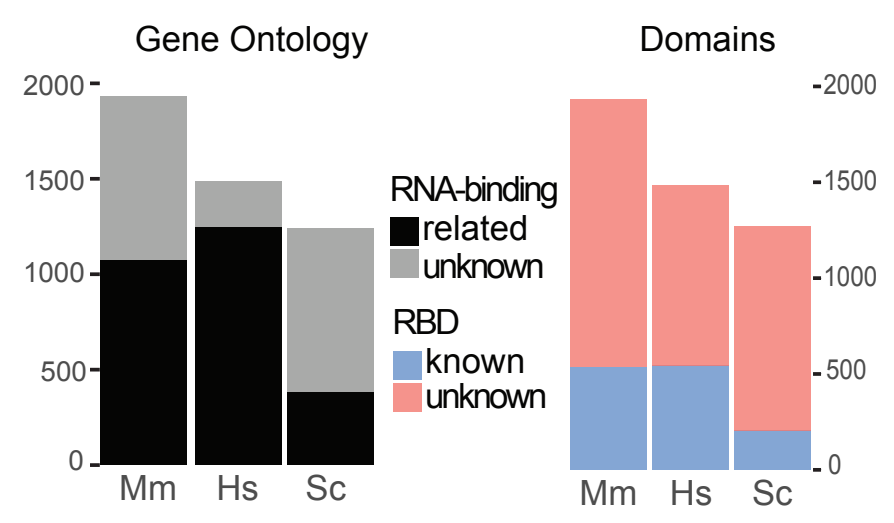

C Overlap human superset with SONAR

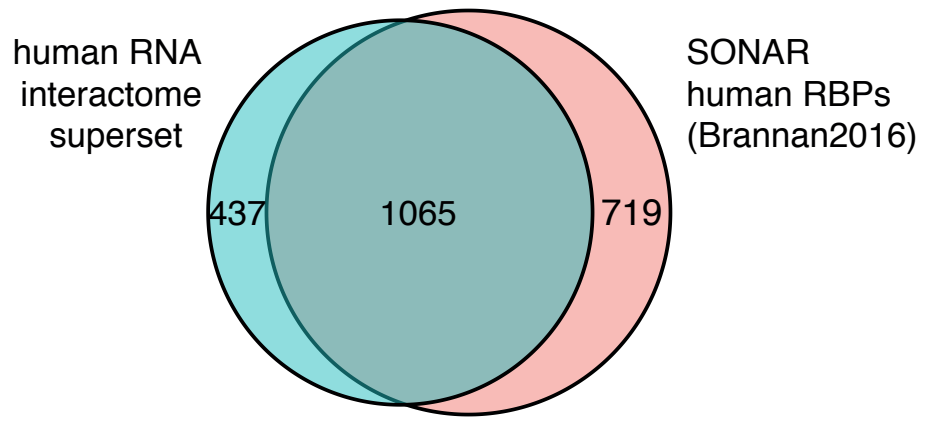

b Overlap of human superset with Gerstberger2014 (Total 2169)

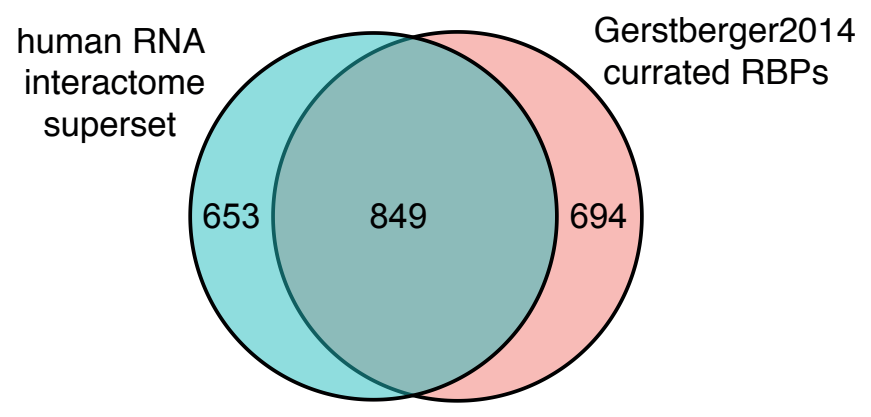

d Yeast RNA interactome superset vs. SONAR and protein array

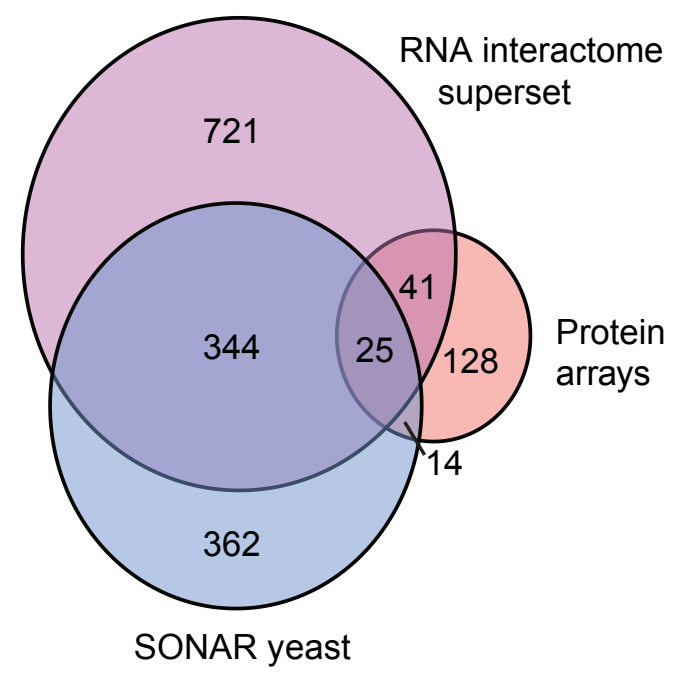

